\title{
Frontal but not parietal cortex is required for decisions under risk
}

Xiaoyue Zhu ${ }^{1,2}$, Joshua Moller-Mara ${ }^{1,2}$, Sylvain Dubroqua ${ }^{1,2}$, Chaofei Bao ${ }^{1,2}$, Jeffrey

C. Erlich ${ }^{1,2,3^{*}}$

1 NYU-ECNU Institute of Brain and Cognitive Science at NYU Shanghai, China

2 NYU Shanghai, Shanghai, China

3 Shanghai Key Laboratory of Brain Functional Genomics (Ministry of

Education), East China Normal University, Shanghai, China

* jerlich@nyu.edu

\section{Abstract}

Neurons in frontal and parietal cortex encode task variables during decision-making, but causal manipulations of the two regions produce strikingly different results. For example, silencing the posterior parietal cortex (PPC) in rats and monkeys produces minimal effects in perceptual decisions requiring integration of sensory evidence, but silencing frontal cortex profoundly impairs the same decisions. Here, we tested, for the first time, the causal roles of the rat frontal orienting field (FOF) and PPC in economic choice under risk. On each trial, rats chose between a lottery and a small but guaranteed surebet. The magnitude of the lottery was independently varied across trials and was indicated to the rat by the pitch of an auditory cue. As in perceptual decisions, both unilateral and bilateral PPC muscimol inactivations produced minimal effects. FOF inactivations produced substantial changes in behavior even though our task had no working memory component. We quantified control and bilateral inactivation behavior with a multi-agent model consisting of a mixture of a 'rational' utility-maximizing agent $\left(U=V^{\rho}\right)$ with two 'habitual' agents that either choose surebet or lottery. Silencing PPC produced no significant shifts in any parameters relative to controls. Effects of FOF silencing were best explained by a decrease in $\rho$, the exponent of the utility function. This effect was parsimoniously explained by a dynamical model where the FOF is part of network that performs sensory-to-value transformations.

\section{Introduction}

Understanding decisions under risk is of substantial interest from a public health and welfare perspective: excessive risk-taking is associated with drug and gambling addiction (Ahmed, 2018), dangerous teen driving (Williams, 2003) and other pathologies (Clifton et al., 2018). On the other hand, inadequate risk-taking is also undesirable: people who avoid investing in the stock market can have their savings diminished by inflation; a mouse that is unwilling to risk predation for foraging will starve. Data from twin and genome-wide association studies (23and Me Research Team et al., 2019, Anokhin et al., 2009, Rao et al., 2018, Xuan et al., 2017) suggest that genetics accounts for a moderate proportion $(\sim 30 \%)$ of variation in 
risk-tolerance, indicating that animal models can help establish the link between genes, brains and risk-tolerance.

The neurobiology of risky decision-making has been studied in human, non-human primate and rodent subjects (reviewed in Orsini et al., 2015, Padoa-Schioppa, 2011, Platt and Huettel, 2008). Rodent work has mostly focused on decision-making under uncertainty in new or changing environments: that is, the neural mechanisms for learning the values of actions. Action-value learning under 'unexpected uncertainty' has also been studied extensively in monkeys and humans (Behrens et al., 2007, Monosov et al., 2015, O'Doherty et al., 2015). These studies typically identify regions that are associated with learning action-values in general: amygdala (Larkin et al., 2016, Orsini et al., 2017, Saez et al., 2017), basal ganglia (Floresco et al., 2018, Samejima et al., 2005, Stopper et al., 2013), and orbital and medial prefrontal cortex (Ogawa et al., 2013, Stopper et al., 2012, van Holstein and Floresco, 2020). Human, monkey and, to a lesser extent, rodent work has also examined the neurobiology of decision-making under risk when the probabilities are known, i.e. 'expected uncertainty' (Christopoulos et al., 2009, Constantinople et al., 2019b, Hocker et al., 2021, Kobayashi and Hsu, 2017, 2019). This is closer to the way risky decisions are studied in economics or finance research, where the potential outcomes of different actions are given explicitly on each trial. In these studies, activity in regions associated with orienting decisions (Hanks et al., 2015) including the parietal cortex (Dorris and Glimcher, 2004, Platt and Glimcher, 1999, Platt and Huettel, 2008) and frontal cortex (Chen and Stuphorn, 2015, 2018) represents the value of the options, as the subjects were typically asked to respond by shifting gaze to a spatial target.

One challenge in synthesizing the vast literature on decisions under risk is that risk-tolerance is not monolithic (Yates, 1992). When behavior is measured either in the laboratory or in real-life, any avoidance of uncertainty can be considered as 'risk-aversion', but such avoidance can come from distinct cognitive constructs. For example, risky behavior in teenagers may result from an incomplete perception of risk associated with those behaviors rather than a greater tolerance for the actual risk (Cohn et al., 1995). Reinforcement learning agents with high learning rates will seem more risk-averse because they will avoid actions after a single loss (i.e. 'lose-shift'), even if on average that action provides good outcomes (March, 1996, Niv et al., 2002). Under the expected utility framework, risk-aversion is usually associated with a decelerating utility function: the more rapid the deceleration, the more risk-averse the subject (Kahneman and Tversky, 1979, Rabin and Thaler, 2001, Von Neumann and Morgenstern, 1953). In finance, risk-aversion is typically modeled as variance-aversion (Duxbury and Summers, 2004). This rich taxonomy of constructs underlying risk-preference not only adds confusion when parsing the literature, but also makes the design of animal experiments estimating all elements simultaneously difficult.

Here, we present results from a risky choice task where the animals make choices under 'expected uncertainty' on a trial-by-trial basis. On each trial, the rat makes an informed decision between a 'surebet' (small but guaranteed reward) and a lottery with fixed probability and cue-guided magnitude. Our model-based quantification of animals' behavior incorporates parameters to capture marginal utility, decision noise, and choice biases. This task and modeling framework provides a foundation for rigorous circuit level exploration of the neurobiology of risky choice. With this framework, we examined the causal contribution of the frontal orienting field (FOF) in frontal cortex and the posterior parietal cortex (PPC): two cortical areas that have been implicated in perceptual decision-making (Erlich et al., 2015, Hanks et al., 2015, Raposo et al., 2014, Zhong et al., 2019).

In perceptual decision that require working-memory, the FOF seems to be essential for maintaining a plan of the upcoming choice. Unilateral silencing of FOF biases 
animals towards the ipsilateral choice and this bias is larger for trials with longer memory periods (Piet et al., 2017). Bilateral silencing also generates an impairment that grows with longer delays or periods of integration (Erlich et al., 2015). In order to distinguish the role of working-memory from the cognitive processes required for economic choice under risk (i.e. trading off the cost of uncertainty with the benefits of a larger reward), we did not include a working-memory component in our task.

Nonetheless, we predicted that unilateral silencing of FOF would cause contralateral impairments in economic choices, due to our hypothesis that FOF serves as a bottleneck for higher order cognitive processes to guide orienting decisions. For bilateral FOF the prediction was less clear: we expected it to influence behavior, possibly by increasing the decision noise.

In contrast to the results from FOF, we previously found that unilateral silencing of PPC in rats did not bias perceptual decisions. PPC only biased 'free-choice' trials, where the animal was rewarded regardless of the left or right response (Erlich et al., 2015). We speculated that the difference between the efficacy of PPC inactivations in perceptual vs. free choice might be that PPC only plays a causal role when decisions are internally guided. Risky choices are internally guided in the sense that each subject has some risk-preference: there is no single 'correct answer' on each trial. Moreover, signatures of expected value are reliably found in PPC (Dorris and Glimcher, 2004, Platt and Glimcher, 1999). Thus, we hypothesized that PPC silencing might influence economic choices, in contract to perceptual decisions.

Although there is substantial literature comparing different functional forms of decision under risk (Farashahi et al., 2019, Heilbronner, 2017, Spitmaan et al., 2019), we are unaware of any previous studies that simultaneously estimates these parameters, examines how silencing of frontal or parietal cortices shifts specific cognitive constructs underlying risky choice. We found that PPC silencing had minimal effects on decisions under risk (but biased free choice). Surprisingly, we found that bilateral silencing of FOF shifted animals away from choosing the lottery. Model-based analysis of these results indicated that the shift was likely caused by a change in the utility function. Moreover, this effect can be parsimoniously explained by a dynamical model where the FOF part of a network for encoding the value of the lottery.

\section{Materials and Methods}

\section{Subjects}

Six male Sprague Dawley rats and two male Brown Norway rats (Vital River, Beijing, China) were used in this study. Rats were placed on a controlled-water schedule and had access to free water 20 minutes each day in addition to the water they earned in the task. Rats were kept on a reversed 12 hour light-dark cycle and were trained during their dark cycle. Animal use procedures were approved by New York University Shanghai International Animal Care and Use Committee following both US and Chinese regulations.

\section{Behavioral Apparatus}

Animal training took place in custom behavioral chambers, located inside sound- and light-attenuated boxes. Each chamber $(23 \times 23 \times 23 \mathrm{~cm})$ was fit with 8 nose ports arranged in four rows (FIGURE 1A), with speakers located on the left and right side. Each nose port contained a pair of blue and a pair of yellow light emitting diodes (LED) for delivering visual stimuli, as well as an infrared LED and infrared 
phototransistor for detecting rats' interactions with the port. The port in the bottom ${ }_{110}$ row contained a stainless steel tube for delivering water rewards. Each training session 111 lasted for 90 minutes.

\section{Behavior}

Trials began with both yellow and blue LED turning on in the center port. This cued the animal to poke its nose into the center port and hold it there for $1 \mathrm{~s}$, after which the center lights were turned off and the choice ports became illuminated. We refer to this period as the 'soft fixation' period, as the animal was allowed to withdraw any time after the initial poke. From here, if the animal poked into a different port other than the center port, a short white noise would play to indicate that this is a mistake. The choice ports will be triggered as long as the animal performed a second poke into the center port. All animals exploited the soft fixation strategy, albeit to different degrees individually. They tended to withdraw after the initial poke but stayed close to the center port during the soft fixation period (FIGURE S2B).

During the soft fixation period a tone played from both speakers, indicating the lottery magnitude for that trial. There were 6 distinct frequencies indicating different lottery magnitudes $(2.5 \mathrm{kHz}-20 \mathrm{kHz}, 75 \mathrm{~dB})$, and all rats had a positive frequency-to-magnitude mapping. The frequency of each lottery was around one octave away from the adjacent tones, making distinguishing the different offers perceptually easy (Dent et al., 2018). For all animals except for subject 2160, the surebet port was on the left and the lottery port was on the right. At the end of fixation, the lottery port and surebet port were illuminated with yellow and blue lights, respectively. The tone stopped as soon as the animal made a choice by poking into one of the choice ports. If the animal chose surebet, a small and guaranteed reward would be delivered at the reward port. If the animal chose lottery, it would either receive the corresponding lottery magnitude or nothing based on the lottery probability, which was titrated for an animal and ranged from 0.5 to 0.6 across all subjects. We refer to these trials as 'choice' trials. In order to ensure that the subjects experienced all the outcomes, the choice trials were randomly interleaved with trials that we refer to as 'forced' trials. The forced trials differ from choice trials in that only one of the two ports was illuminated and available for poking, forcing the animal to make that response. The forced surebet and forced lottery trials together accounted for $25 \%$ of the total trials. The inter-trial intervals (ITI) were between 3 and 10 seconds. A trial was considered a violation if the animal failed to poke into the center port within 300 seconds from trial start, or it did not make a choice 30 seconds after fixation. Violations were excluded from all analyses, except where they are specifically mentioned.

In some sessions, 'free' trials were interleaved with the choice and forced trials. Free trials were similar to choice trials except at the end of fixation both left and right port were illuminated with blue LEDs. The animal would receive a medium-sized reward ( 2 times the surebet) regardless of which port it chose. The free trials were randomly interleaved with the choice and forced trials, and were introduced only after all the experiments presented in FIGURE 1-7 were completed.

Training pipeline Animal training took place in two distinct phases: the operant conditioning phase and the risky choice phase. Briefly, in the operant conditioning phase, rats became familiar with the training apparatus and learned to poke into the reward port when illuminated. Trials began with the illumination of the reward port, and water reward was immediately delivered upon port entry. After the rats learned to poke in the reward port reliably, they proceeded to the next training stage where 
they had to first poke into an illuminated choice port (left or right, chosen randomly) before the reward port was illuminated for reward. They graduated to the risky choice phase if they correctly performed these trials at least $40 \%$ of the session.

In the risky choice phase, rats started with only two frequencies: the lowest and highest, corresponding to the smallest and largest lottery magnitude. Initially, there were more forced trials than choice trials to help them understand the task. Once the animals reliably differentiated between the low and high lottery choice trials, more choice trials were added. The intermediate frequencies were added one by one, contingent upon good behavior in the choice trials with existing frequencies. The lottery probability and the surebet magnitude were adapted to each animal so that their preferences could be reliably estimated. For example, if an animal chose the lottery too often, the lottery probability would be decreased.

\section{Surgery}

Surgical methods were similar to those described in Erlich et al. (2015). The rats were anesthetized with isoflurane and placed in a stereotaxic apparatus (RWD Life Science Co.,LTD, Shenzhen). The scalp was shaved, washed with ethanol and iodopovidone and incised. Then, the skull was cleaned of tissue and blood. The stereotax was used to mark the locations of craniotomies for the left and right FOF and PPC, relative to Bregma on the skull. Four craniotomies and durotomies were performed and the skull was coated with a thin layer of C\&B Metabond (Parkell Inc., NY). Guide cannula along with the injector (RWD Life Science Co.,LTD, Shenzhen) was inserted $1.5 \mathrm{~mm}$ into the cortex measured from brain surface for each craniotomy. The guide cannulae were placed and secured to the skull one at a time with a small amount of Absolute Dentin (Parkell Inc., NY). The injector was removed from each guide once the guide was secured to the skull. After all four guide cannulae were in place, more Absolute Dentin was applied to cover the skull and further secure the guide cannulae. Vetbond (3M, U.S.) was applied to glue the surrounding tissue to Absolute Dentin. The animals were given 7 days to recover on free water before resuming training.

\section{Cannulae}

All 8 rats were implanted bilaterally in FOF ( $+2 \mathrm{AP}, \pm 1.5 \mathrm{ML} \mathrm{mm}$ from Bregma) with 26 AWG guide cannulae (RWD Life Science Co.,LTD, Shenzhen) and in lateral PPC (-3.8 AP, $\pm 3.0 \mathrm{ML}$ from Bregma) with 26 AWG guide cannulae (4 cannulae per rat total). The tip of the guide sat on the brain surface, while the 33 AWG injector was extended $1.5 \mathrm{~mm}$ below the bottom of the guide cannula. The dummy extended $0.5 \mathrm{~mm}$ into the cortex.

\section{Infusions}

Infusions were performed once a week with normal training days taking place on all other days. This was to minimize adaptation to the effects of the muscimol and to have stable performance in the sessions immediately before infusion sessions. Animals were held by an experimenter during the infusion, no general anesthetic was administered. On an infusion day, the rat was placed on the experimenter's lap and the dummy cannulae were gently removed and cleaned with iodine and alcohol. The injector was inserted into the target guide cannula and reached $1.5 \mathrm{~mm}$ into cortex. A $1 \mu \mathrm{L}$ syringe (Gaoge, Shanghai) connected via tubing filled with mineral oil to the injector was used to infuse $0.3 \mu \mathrm{L}$ of muscimol (of various concentrations) into cortex. The injection was done over 1 minute, after which the injector was left in the brain for 5 more minutes to allow diffusion before removal. The thoroughly cleaned dummies were placed into the 
guide cannula. The rats began training 2 to 53 minutes after the infusion, the average ${ }_{206}$ time between infusion and starting of the behavioral session was 27 minutes. See FiguRE S1 for the complete list of all infusion doses, regions, and order for each rat.

\section{Analysis}

For all analyses, we excluded time out violation trials (where the subjects disengaged from the ports for more than $30 \mathrm{~s}$ during the trial) and trials with reaction time longer than 3 s. Unless otherwise specified, the 'control' sessions refer to the sessions one day before any infusion event during the course of the experiment. All analysis and statistics were computed in $\mathrm{R}$ (version 3.6.3, R Foundation for Statistical Computing, Vienna, Austria), except for the biophysical model, which was simulated in Julia (1.6.0, Bezanson et al., 2017).

Generalized linear mixed-effects models (GLMM) GLMMs were fit using the lme4 R package (Bates et al., 2015). To test whether bilateral infusion had any effects on performance, we specified a mixed-effects model where the probability of a lottery choice was a logistic function of $E V_{\text {lottery }}-E V_{\text {surebet }}$, muscimol dosage $(\mu \mathrm{g})$ and their interaction as fixed effects. The rat and an interaction of rat, $E V_{\text {lottery }}-E V_{\text {surebet }}$ and dosage were modelled as within-subject random effects. The expected value of lottery is the product of the lottery magnitude and lottery probability $\left(E V_{\text {lottery }}=P_{\text {lottery }} \cdot V_{\text {lottery }}\right)$. Similarly, $E V_{\text {surebet }}$ denotes the expected value of surebet, which is simply the value of surebet here $\left(E V_{\text {surebet }}=V_{\text {surebet }}\right.$, since $\left.P_{\text {surebet }}=1\right)$. In standard $\mathrm{R}$ formula syntax:

$$
\text { chose_lottery } \sim \text { delta_EV } * \text { dosage }+(\text { delta_EV } * \text { dosage } \mid \text { subjid })
$$

where chose_lottery is 1 if lottery was chosen on a trial, delta_EV is $E V_{\text {lottery }}-E V_{\text {surebet }}$ and subjid is the subject ID for each rat.

To test whether unilateral infusions caused a left/right bias (as in Erlich et al., 2015), we specified a mixed-effects model similar to the one described above:

chose_right $\sim$ rl_delta_EV $*$ infusion_side $+(r$ _delta_EV $*$ infusion_side|subjid $)$

where chose_right is 1 if the right port is chosen on this trial, rl_delta_EV is $E V_{\text {right }}-E V_{\text {left }}$ and infusion_side is a categorical variable with three levels: left, right and control. The plots in Figure S4 (FOF) and Figure S5 (PPC) show that the model fits for each rat are good, reflecting how the random effects allow for each rats' data to be fit, while also finding significant fixed effects.

To estimate the shift in indifference point induced by bilateral FOF inactivation, we first fit a GLMM as described above. We generated synthetic data points for delta_EV to extend its range, and the model was used to predict $p$ (Choose Lottery) for each synthetic data point. For each animal, we identified the delta_EV values that resulted in $p$ (Choose Lottery) to be between 0.499 and 0.501 , which is the definition of indifference point. The average indifference point was obtained by taking the mean of such values across animals.

To test whether unilateral PPC infusions led to an ipsilateral bias in both free choice and risk choice trials, we specified a GLMM as following:

$$
\text { chose_ipsi } \sim \text { infusion }+(\text { infusion|subjid })
$$


where chose_ipsi is a binary variable indicating whether the animal chose the side ipsilateral to the infusion side or not, and infusion is a binary variable representing the presence of an unilateral PPC infusion.

To estimate changes in reaction time, we used Linear Mixed-Effects Models (LMM). The formula for bilateral infusion is:

$$
\log (\mathrm{RT}) \sim \text { delta_EV } * \text { dosage } * \text { choice }+(\text { delta_EV } * \text { dosage } * \text { choice } \mid \text { subjid })
$$

where $\log (\mathrm{RT})$ denotes the logarithm of reaction time, choice is a binary value for the surebet/lottery choice (0/1). Similarly, the formula for unilateral infusion is:

$\log (\mathrm{RT}) \sim$ rl_delta_EV $*$ infusion_side $*$ choice $+($ rl_delta_EV $*$ infusion_side $*$ choice $\mid$ subjid $)$

To test whether the outcome of the previous trial affected choice on the current trial, we first classified the previous trial's outcome into three categories: lottery-win, lottery-lose and surebet. If the previous trial was a violation, we considered that as a surebet choice. A mixed-effects model was specified:

$$
\text { chose_lottery } \sim \text { delta_ev }+ \text { prev_outcome }+(\text { delta_ev }+ \text { prev_outcome|subjid })
$$

where prev_outcome is a categorical variable with three levels of previous outcome as above.

The three-agent mixture model We developed a three-agent mixture model that used 4 parameters to transform the offers on each trial into a probability of choosing lottery as a weighted outcome of three agents (FIGURE 4A): a rational agent, a 'lottery agent' and a 'surebet' agent. For the rational agent, we assume an exponential term $\rho$ for the utility function, $U=V^{\rho}$. A concave utility function $(\rho<1)$ implies risk aversion, a linear function with $\rho=1$ implies being risk-neutral and a convex function $(\rho>1)$ implies risk seeking. We modeled noise in the rational agent, $\sigma$, as the standard deviation in the expected utility distribution. This noise term represents the idea that animals' internal representation of utility is noisy. The source of the noise could be neural noise or uncertainty in learning the map between perceptual stimuli and lottery magnitudes. Although perceptual noise could also play a role, we think the contribution would be small since the lottery tones are spaced around 1 octave apart from each other, which is easily distinguishable for rats (Dent et al., 2018). Concretely,

$$
\begin{aligned}
E U_{L} & \sim \mathcal{N}\left(V_{L}^{\rho} P_{L}, \sigma\right) \\
U_{S B} & \sim \mathcal{N}\left(V_{S B}^{\rho}, \sigma\right)
\end{aligned}
$$

where the expected utility of lottery, $E U_{L}$, and the utility of the surebet, $U_{S B}$ are Normal distributions. $V_{L}, V_{S B}$ refer to the magnitude of lottery and surebet and $P_{L}$ is the probability of lottery payout. The probability of choosing lottery for the rational agent then becomes

$$
\begin{aligned}
p_{\text {Choose Lottery }}^{\text {rational }} & =p\left(E U_{L}>U_{S B}\right) \\
& =p\left(E U_{L}-U_{S B}>0\right) \\
& =p\left(\mathcal{N}\left(V_{L}^{\rho} P_{L}, \sigma\right)-\mathcal{N}\left(V_{S B}^{\rho}, \sigma\right)>0\right) \\
& =p\left(\mathcal{N}\left(V_{L}^{\rho} P_{L}-V_{S B}^{\rho}, \sqrt{2} \sigma\right)>0\right) \\
& =1-\Phi\left(0 ; V_{L}^{\rho} P_{L}-V_{S B}^{\rho}, \sqrt{2} \sigma\right)
\end{aligned}
$$


where $\Phi\left(0 ; V_{L}^{\rho} P_{L}-V_{S B}^{\rho}, \sqrt{2} \sigma\right)$ is the cumulative Normal distribution with mean $V_{L}^{\rho} P_{L}-V_{S B}^{\rho}$, standard deviation $\sqrt{2} \sigma$ and evaluated at 0 . Note that this provides fits with similar likelihood as the softmax choice function with $\beta$ as temperature:

$$
\begin{aligned}
\Delta E U & =V_{L}^{\rho} P_{L}-V_{S B}^{\rho} \\
p(\text { Choose Lottery }) & =\frac{1}{1+e^{-\beta \Delta E U}}
\end{aligned}
$$

The other two agents in the three-agent mixture model are the lottery and surebet agents. They represent the habitual bias of the animal to make one or the other choice regardless of the lottery offer, similar to biased lapse terms in Erlich et al. (2015). The probability of choosing lottery for the lottery agent is $p_{\text {Choose Lottery }}^{\text {lottery }}=1$ and for the surebet agent is $p_{\text {Choose Lottery }}^{\text {surebet }}=0$.

The last step is to obtain $p$ (Choose Lottery) by mixing the probability from each agent $\vec{P}$ with their respective mixing weights $\vec{\omega}$ that sum up to 1 . Formally,

$$
\begin{aligned}
P(\text { Choose Lottery }) & =\vec{P} \cdot \vec{\omega} \\
& =P_{\text {Choose Lottery }}^{\text {rational }} \omega_{\text {rational }}+1 \cdot \omega_{\text {lottery }}+0 \cdot \omega_{\text {surebet }} \\
\sum \vec{\omega} & =1
\end{aligned}
$$

Model fitting Following modern statistical convention, we estimated the posterior distribution over model parameters with weakly informative priors using the rstan package (v2.21.2; Stan Development Team, 2020). rstan is the $\mathrm{R}$ interface of Stan (Stan Development Team, 2020), a probabilistic programming language that implements Hamiltonian Monte Carlo (HMC) algorithm for Bayesian inference. The prior over the utility exponent $\rho$ was Lognormal $(\log (0.9), 0.4)$, a weakly informative prior that prefers $\rho$ to be close to risk-neutral. The prior over noise $\sigma$ was $\operatorname{Gamma}(6,3)$. The prior over the mixing weights $\vec{\omega}$ was a Dirichlet distribution with the concentration parameter $\alpha=[6,2,2]$. The resulting $\omega_{\text {rational }}$ distribution was broad and had the mean of 0.6 , both $\omega_{\text {lottery }}$ and $\omega_{\text {surebet }}$ distribution had the mean of 0.2. By attributing more weight to the rational agent over the habitual agents, the prior reflected our selection of the experimental animals - only the ones with good psychometric curves were included. Four Markov chains with 1000 samples each were obtained for each model parameter after 1000 warm-up samples. The $\hat{R}$ convergence diagnostic for each parameter was close to 1 , indicating the chains mixed well.

Inactivation mixture model We constructed a different version of the three-agent model, which considered two datasets from each rat simultaneously: an inactivation (bilateral FOF) and a control dataset. The model's raw parameters included $\rho_{\text {base }}$, a parameter for $\rho$ in the $\log$ space, its prior was $\operatorname{Lognormal}(\log (0.9), 0.4) ; \sigma_{\text {control }}$, as $\sigma$ in the original version, with a prior of $\operatorname{Gamma}(6,3) ; \omega_{1}$, with a prior of $\mathcal{N}(0,2)$, equivalent to $\omega_{\text {rational }}$ after a logistic transformation; and $\omega_{2}$ with a prior of $\mathcal{N}(0,2)$, representing the proportion of the surebet agent in $1-\operatorname{logistic}\left(\omega_{1}\right)$ after the logistic transformation itself, where $\operatorname{logistic}(x)=1 /\left(1+e^{-x}\right)$ : 


$$
\begin{aligned}
\rho & =e^{\rho_{\text {base }}} \\
\sigma & =\sigma_{\text {control }} \\
\omega_{\text {rational }} & =\operatorname{logistic}\left(\omega_{1}\right) \\
\omega_{\text {surebet }} & =\left(1-\omega_{\text {rational }}\right) \cdot \operatorname{logistic}\left(\omega_{2}\right) \\
\omega_{\text {lottery }} & =\left(1-\omega_{\text {rational }}\right) \cdot\left(1-\operatorname{logistic}\left(\omega_{2}\right)\right)
\end{aligned}
$$

For the inactivation dataset, we added a new parameter for each raw parameter in order to estimate the effects of inactivation:

$$
\begin{aligned}
\rho & =e^{\rho_{\text {base }}+\Delta \rho_{\text {base }}} \\
\sigma & =\sigma_{\text {control }} \cdot \text { sigma } \\
& \text { logistic }\left(\omega_{1}+\Delta \omega_{1}\right) \\
\omega_{\text {rational }} & =\left(1-\omega_{\text {rational }}\right) \cdot \operatorname{logistic}\left(\omega_{2}+\Delta \omega_{2}\right) \\
\omega_{\text {surebet }} & =\left(1-\omega_{\text {rational }}\right) \cdot\left(1-\operatorname{logistic}\left(\omega_{2}+\Delta \omega_{2}\right)\right) \\
\omega_{\text {lottery }} & =(1-{ })
\end{aligned}
$$

where $\Delta \rho_{\text {base }}$ denotes the change in $\rho$ in the log space, it had a prior of $\mathcal{N}(0,1) ; \sigma_{\pi}$, with a prior of $\operatorname{Lognormal}(0,0.1)$, represents how the infusions could scale noise; $\Delta \omega_{1}$ and $\Delta \omega_{2}$ fit potential changes in $\omega_{1}$ and $\omega_{2}$ before the logistic transformation, respectively.

We constructed two other variants of the inactivation model for model comparison. For the $\rho$-only model, both $\Delta \omega_{1}$ and $\Delta \omega_{2}$ were fixed to be 0 during fitting. For the $\omega$-only model, $\Delta \rho_{\text {base }}$ was fixed to be 0 .

Synthetic datasets To test the validity of our model, we created synthetic datasets with parameters generated from the prior distributions described above. The three-agent model was fit to the synthetic datasets, and it was able to recover the generative parameters accurately (FIGURE S8A). This assured that our model can capture the behavior well and has no systematic bias in estimating the parameters.

Mixture model prediction confidence intervals To generate model predictions in between the actual lottery lottery magnitudes (as in FIGURE 4B), we generated a synthetic dataset with narrowly-spaced lottery magnitudes (incremented by 1 ). Then, we sampled parameters from the estimated posteriors and computed the probability of choosing the lottery given the synthetic offers. The resulting output is a $\mathrm{n}_{-}$iter $\times$ n_lott_mag matrix, where $\mathrm{n}_{-}$iter is the number of Markov samples and $\mathrm{n}_{-}$lott_mag is the length of unique lottery magnitudes. Finally, binomial 80\%, 90\%, and 95\% confidence intervals for each lottery offer were estimated by taking the $10 \%$ and $90 \%$, $5 \%$ and $95 \%$, and $2.5 \%$ and $97.5 \%$ percentile of $n_{-}$iter predicted choices, respectively.

Mixture model comparison To understand which model describes the inactivation results best, we performed 10-fold cross-validation of the MCMC fits of each model. For each fold, the model first fit on the training data, containing $90 \%$ of the original data from each condition (control and bilateral FOF). We then computed (in the generated quantities block) the log predictive densities by passing in the held-out data, using the posterior draws conditional on the training data. As the training and testing data are independent, the log predictive density coincides with 
the log likelihood of the test data. To evaluate the predictive performance, we computed the expected log pointwise predictive density (ELPD) using the test data (Vehtari et al., 2017). As the definition of ELPD incorporates the true generating process of prediction that is unknown, in practice, ELPD is approximated by computing the log predictive density using draws from the posterior samples

$$
\widehat{l p d}=\sum_{i=1}^{n} \log \left(\frac{1}{S} \sum_{s=1}^{S} p\left(y_{i} \mid \theta^{s}\right)\right),
$$

where $n$ is the number of test trials, $\theta^{s}$ is the s-th parameter sample from the posterior, and $p\left(y_{i} \mid \theta^{s}\right)$ is the log predictive density of the i-th test trial computed using the s-th parameter sample. Intuitively, the closer ELPD is to 0, the higher the model predictive accuracy.

Biophysical model We generated a 6-node rate model to understand how muscimol inactivation of the FOF could cause a reduction in lottery choices via a change in the curvature of the utility function. The activity of the six nodes, $X$, are governed by the following equations, where $v$ is the magnitude of the lottery and the $i$ in $g(v, t, i)$ represents the node index (1-6). Simulation was done using Euler's method in Julia (1.6.0, Bezanson et al., 2017):

$$
\begin{aligned}
d X & =d t(-X / \tau+W X+g(v, t, i)+\mathcal{N}(0, \sigma)) \\
X & =f(X+d X) \\
f(x) & = \begin{cases}x & \text { if } 0 \leq x \leq 100 \\
0 & \text { if } x<0 \\
100 & \text { if } x>100\end{cases} \\
g(v, t, i) & = \begin{cases}0 & \text { if } 0.1>t>1 \text { s or } i \leq 3 \\
v\end{cases} \\
\tau & =0.15 \mathrm{~s} \\
d t & =0.001 \mathrm{~s} \\
\sigma & =\frac{5}{6} \cdot d t^{-1} \\
\text { for } w_{i j} \in W & \\
w_{i j} & \sim \mathcal{N}(5 / 6,0)
\end{aligned}
$$

We began the simulation of each trial a few seconds before the input was turned on, to allow the network to reach its baseline fixed-point. We examined different instantiations of this model by generating the weight matrix, $W$, from different random seeds. Many (but not all) of these networks gave qualitatively similar results.

\section{Surebet learning}

To test the role of PPC in learning, we periodically changed the surebet magnitude in a model-based way to shift the decision boundary. For each shift, we fit the three-agent model on control data from the past 14 days to obtain a set of parameters. Using a binary search algorithm, we then used those parameters to generate synthetic choices with different surebet magnitudes until we found a value that produces a shift in overall probability choosing lottery $(p$ (Choose Lottery $))$ close to the target (drawn 
uniformly from $\pm U(0.2,0.3))$. The new surebet magnitude was assigned to the animal on the day of change. All animals in the surebet learning experiment had undergone two rounds of shift without any infusion, in the course of 14 days, to acclimate them to the new routine before bilateral PPC infusions. The first two surebet change sessions are not included in the analysis of FiguRE 7. 


\section{Results}

\section{Behavior}

In this paper, we only present behavior from sessions after the animal was implanted with cannulae for experiments. Unless otherwise specified, control sessions were the sessions from the day before the infusion sessions. The animals' choices were consistent with a utility-maximizing strategy: they had relatively few violations of first-order stochastic dominance (i.e. they chose the surebet when the lottery magnitude was less than the surebet magnitude) and they increased the proportion of lottery choices monotonically with increasing expected value (FIGURE 1B-D). Six of the rats were risk-averse and two were close to risk-neutral (FIGURE 1D). On average, each rat completed 82 choices in a control session (FIgURE 1E).

\section{Effects of silencing FOF and PPC on the risky choice task}

All animals experienced three different types of inactivations (left, right and bilateral) in two brain areas (FOF \& PPC). In total, we include 7456 choice trials from 127 infusions sessions into the FOF and PPC of 8 rats. The details of region, order and dosage of the infusions for each rat is shown in FIgURE S1.

\section{FOF silencing shifted choices away from the lottery}

Bilateral silencing of the FOF (FIGURE 2A) resulted in a dose-dependent reduction in lottery choices (FIgURE 2B; for individual subjects see Figure S4A). A

generalized-linear mixed-effects model (GLMM) of the bilateral infusions found a significant main effect of muscimol dosage $\left(\beta_{\text {dose }}=-3.18 \pm 0.92, p<0.01\right)$. The mean indifference point (in units of $E V_{\text {lottery }}-E V_{\text {surebet }}=\mu L$ of water) shifted from 50.92 \pm 11.56 in control to $154.43 \pm 23.49$ under $0.3 \mu \mathrm{g}$ muscimol $\left(T_{8}=-3.95, p<0.001\right)$. In other words, inactivating bilateral FOF is equivalent to adding around $100 \mu \mathrm{L}$ to the surebet. There also was a small but significant decrease in the slope of the logistic curve $\left(\beta_{E V_{\text {lottery }}-E V_{\text {surebet }}: \text { dose }}=-0.08 \pm 0.02, p<0.001\right)$. Bilateral silencing of the FOF did not consistently change animal's reaction time, defined as the time from center port withdrawl until a choice port poke (Linear mixed-effects model, LMM, $\left.\beta_{\text {dose }}=0.21 \pm 0.29, p=0.574\right)$. However, there was a significant slowing effect in three animals: $2152\left(\beta_{\text {dose }}=2.33 \pm 0.42, p<0.001\right), 2153\left(\beta_{\text {dose }}=1.31 \pm 0.39, p<0.01\right)$ and $2166\left(\beta_{\text {dose }}=1.22 \pm 0.25, p<0.001\right)$, possibly due to muscimol spillover into the adjacent M1 area (FIGURE S3C). Overall, the slowing effect from bilateral FOF inactivation was less reliable across animals than the effect on choice (FIGURE S6A), suggesting the effect on choice was not primarily driven by changes in movement.

Unilateral infusions had a smaller effect compared to bilateral infusions (FIGURE $2 \mathrm{C})$. Infusions of $0.3 \mu \mathrm{g}$ muscimol into the left and right FOF resulted in small but significant decrease in the slope of the logistic curve

$\left(\beta_{E V_{\text {right }}-E V_{\text {left }}: \text { left }}=-0.01 \pm 0.003, p<0.001\right.$;

$\left.\beta_{E V_{\text {right }}-E V_{\text {left }}: \text { right }}=-0.01 \pm 0.004, p=0.049\right)$. These results were surprising for two reasons. First, we expected an ipsilateral bias, but both left and right infusions shifted animals slightly to choose leftward choices. As seven out of eight animals had the surebet port assigned on the left, it is possible that the decrease in choosing right after silencing either side of the FOF was, in fact, a partial effect of bilateral FOF inactivation (decrease in choosing the lottery). Second, these effects are very weak compared to the large ipsilateral biases caused by unilateral FOF silencing in previous tasks (Erlich et al., 2011, 2015, Kopec et al., 2015). The discrepancy may be due to the memory component in their tasks, whereas our task does not have one. Overall, 
A

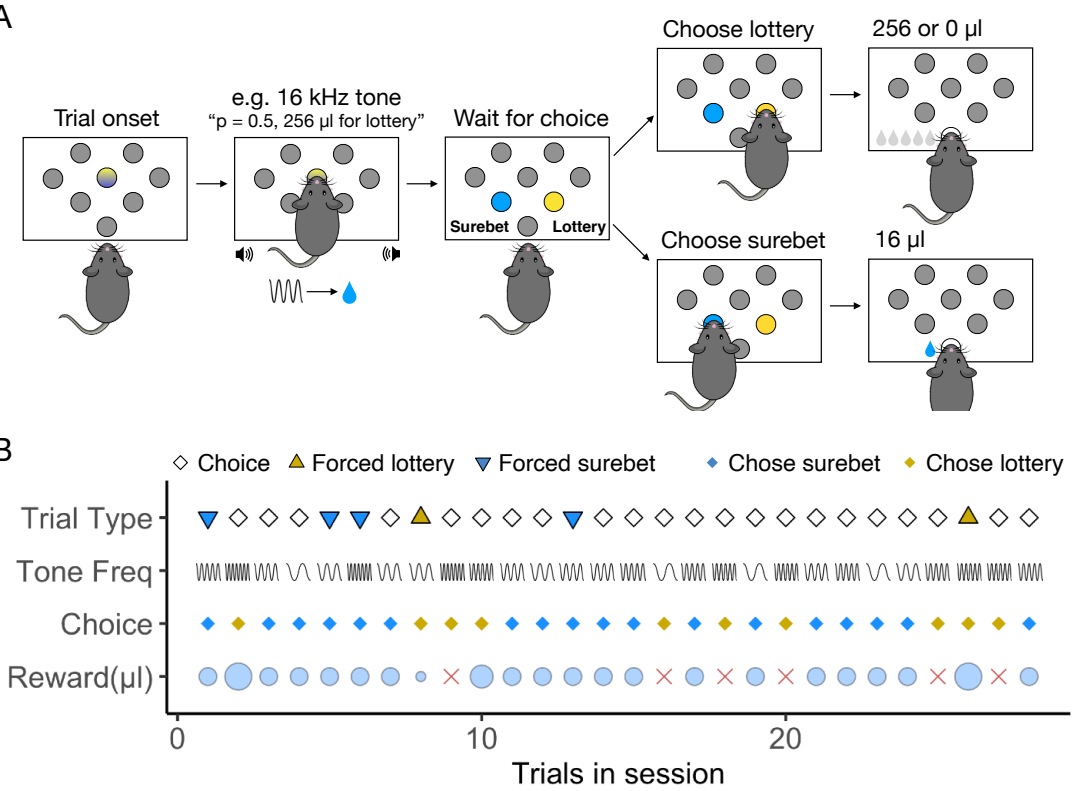

C

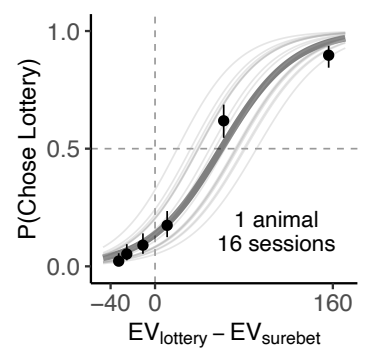

D

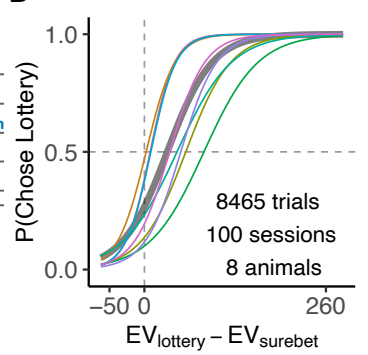

$\mathrm{E}$

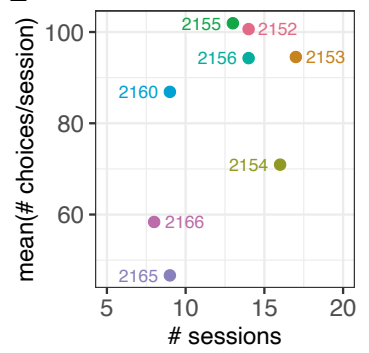

Figure 1. The risky choice task and animal behavior. A. Schematic of the risky choice task. Each trial began with the onset of central LED, which cued the animal to poke into the center port and hold there for $1 \mathrm{~s}$. A tone was played, indicating the magnitude of the lottery. After $1 \mathrm{~s}$, the animal withdrew from the center port and made a choice poke into the surebet or the lottery port. The lottery sound was played until the choice poke. A surebet choice delivered a small and guaranteed reward, whereas a lottery poke gave either 0 or the cued magnitude based on lottery probability, which was fixed for each animal. See more detailed task description in Methods. B. Timeline of trials in one example session. For trial type, white diamond, yellow triangle and blue triangle represents the choice trial, forced lottery trial and forced surebet trial, respectively. The sine-waves in the 'tone freq' row indicate the lottery magnitudes cued by the tone frequency. The more compact the sine-wave is, higher is the lottery magnitude on this trial. Animal's choices are marked in diamonds, with yellow for lottery and blue for surebet. The reward received $(\mu \mathrm{L})$ on each trial is shown in light blue circles, whose size represents the relative amount. The red cross indicates a lost lottery with no rewards. C. Example subject performance from 16 control sessions 1 day before an infusion. The probability of choosing lottery is plotted as as a function of the expected value of lottery minus the expected value of surebet $\left(V_{\text {lottery }} P_{\text {lottery }}-V_{\text {surebet }}\right)$, where $V$ represents $\mu \mathrm{L}$ of water. The circles with error bars are the mean and $95 \%$ binomial confidence intervals. The lines are the psychometric curves generated by a generalized linear model, the thin gray lines are fit to each session, the thick gray line fit to all the sessions combined. D. Subject performance from 100 control sessions 1 day before an infusion event ( 8 rats). The colored lines are the psychometric curves generated by a generalized linear model fit to all the control sessions from each animal, the thick gray line fit to all the sessions combined. E. The number of control sessions within the infusion period, and the average number of choice trials, colored by subject. The text indicates the subject ID. 
unilateral infusions in FOF did not change animal's reaction time (LMM, $\left.\beta_{L}=0.07 \pm 0.07, p=0.44 ; \beta_{R}=0.01 \pm 0.07, p=0.89\right)$.

A

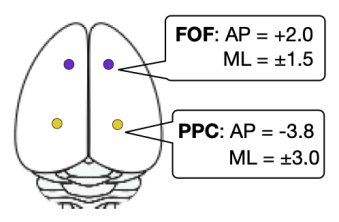

$\mathrm{D}$

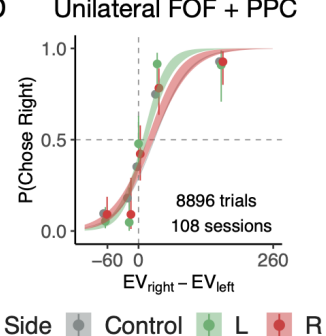

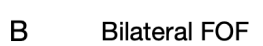

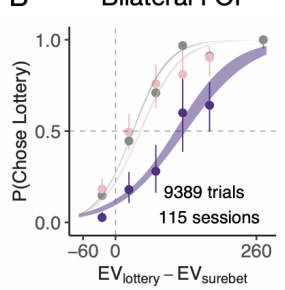

$\operatorname{Dose}(\mu \mathrm{g}) \phi 0 \quad 0.075 \phi 0.3$

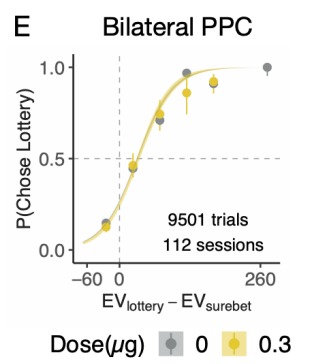

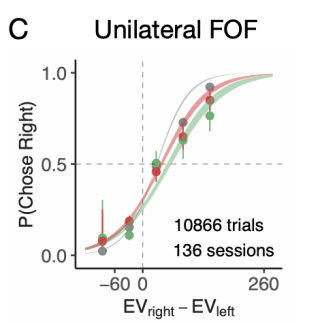

Side $\phi$ Control $\phi \mathrm{L} \phi \mathrm{R}$

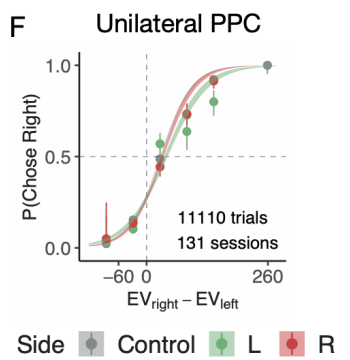

Figure 2. Bilateral and unilateral infusions in FOF and PPC. A. Top-down view of the rat cortex with the target coordinates of FOF and PPC, where the cannulae were implanted. B. Bilateral infusion of muscimol $(0.3 \mu \mathrm{g})$ into the FOF significantly shifted the choices towards the lottery. Control sessions 1 day before an infusion are shown in gray $(\mathrm{n}=17$ sessions, 8 rats), $0.075 \mu \mathrm{g}$ per side bilateral FOF infusions $(\mathrm{n}=6$ sessions, 5 rats $)$ are in light pink, $0.3 \mu \mathrm{g}$ per side bilateral FOF infusions ( $\mathrm{n}=9$ sessions, 8 rats) are in dark purple. The circles with error bars are the mean and $95 \%$ binomial confidence intervals. The ribbons are from a generalized linear model fit to the data. See details in Methods. C. Unilateral infusion of muscimol into the left and right FOF resulted in a small but reliable shift towards surebet. Control sessions are in gray ( $\mathrm{n}=39$ sessions, 8 rats), $0.3 \mu \mathrm{g}$ left FOF infusions ( $\mathrm{n}=16$ sessions, 8 rats $)$ are in green, $0.3 \mu \mathrm{g}$ right FOF infusions $(\mathrm{n}=20$ sessions, 8 rats $)$ are in red. D. Simultaneous unilateral inactivation of FOF and PPC had no effect. Control sessions are in gray ( $\mathrm{n}=8$ sessions, 4 rats), $0.3 \mu \mathrm{g}$ left FOF infusion and $0.6 \mu \mathrm{g}$ left PPC infusions $(\mathrm{n}=4$ sessions) are in green, $0.3 \mu \mathrm{g}$ right $\mathrm{FOF}$ infusion and $0.6 \mu \mathrm{g}$ right $\mathrm{PPC}$ infusion $(\mathrm{n}=4$ sessions $)$ are in red. E. Bilateral infusion of muscimol into the PPC had no effect. Control sessions are in gray ( $\mathrm{n}=24$ sessions, 7 rats), $0.3 \mu \mathrm{g}$ per side bilateral PPC infusions ( $\mathrm{n}=12$ sessions, 7 rats) are in gold. F. Unilateral infusion of muscimol into the PPC had no effect. Control sessions are in gray $(\mathrm{n}$ $=31$ sessions, 8 rats), $0.3 \mu \mathrm{g}$ left $\mathrm{PPC}$ infusions $(\mathrm{n}=11$ sessions, 7 rats, 3$)$ are in green, 0.3 $\mu \mathrm{g}$ right $\mathrm{PPC}$ infusions $(\mathrm{n}=19$ sessions, 8 rats $)$ are in red.

\section{PPC silencing had minimal effect on the risky choices}

Bilateral silencing of PPC resulted in minimal effect on the risky-choice behavior $\left(\beta_{\text {dose }}=-0.60 \pm 0.57, p=0.29\right.$; FigurE $\left.2 \mathrm{E}\right)$. The lack of main effect of dose was found in 5 out of 7 animals (FIGURE S5A), except for 2153

$\left(\beta_{\text {dose }}=-2.12 \pm 0.59, p<0.001\right)$ and $2156\left(\beta_{\text {dose }}=-2.19 \pm 0.84, p<0.01\right)$. To test

for any lateralized effects from unilateral PPC infusions, we performed a second

GLMM test where the choice on each trial was a logistic function of $E V_{\text {right }}-E V_{\text {left }}$, infusion side and their interaction as fixed effects. No significant effects were found on the group level (FIGURE $2 \mathrm{~F}$, all $p>0.5$ ). This was consistent across all subjects except for 2152, 2153 and 2155, left infusion produced a small but significant shift 
away from lottery for these animals (FIguRE S5B). To probe whether perturbation of FOF could reveal an effect of PPC inactivation, we inactivated unilateral FOF (0.3 $\mu \mathrm{g})$ while unilaterally inactivating $\mathrm{PPC}$ with $0.6 \mu \mathrm{g}$ muscimol. The simultaneous inactivation, still, had no significant effect on the behavior (FIGURE 2D, all $p>0.5$ ). Overall, the results suggest that PPC inactivation was ineffective in biasing the risky choices. Thus, our hypothesis that the PPC may be involved in economic decisions because they are an expression of an internal preference was not supported.

In order to establish that our infusions into PPC were effective, after completing all of the experiments reported related to risky-choice, we added a 'free' trial type (as in Erlich et al., 2015). On a free trial, both the surebet port and lottery port were illuminated with blue-LEDs after fixation, accompanied by a brief neutral tone. The animals were rewarded twice the magnitude of the surebet reward regardless of which port they chose (Figure 3A). These types of trials have been demonstrated to be sensitive to unilateral silencing of the PPC (Erlich et al., 2015, Katz et al., 2016). We randomly intermixed $11 \%$ free trials with $22 \%$ forced trials and $67 \%$ choice trials on the control days. After a few sessions with the new trial type, rats expressed a consistent bias on the free trials and still performed the choice trials in a utility-maximizing way. The proportion of free trials was increased to $50 \%$ on the infusion day, with the rest being $12.5 \%$ forced trials and $37.5 \%$ choice trials. Infusions of muscimol $(0.6 \mu \mathrm{g})$ into one hemifield of PPC (opposite to the animal's preferred side) produced a substantial ipsilateral bias on free trials (FIGURE 3B;

$\left.\beta_{\text {infusion }}=1.19 \pm 0.50, p<0.05\right)$. The ipsilateral bias in free trials was observed even while, consistent with our previous PPC inactivation results, there was no ipsilateral bias on the interleaved choice trials (FIGURE 3D; $\beta_{\text {infusion }}=0.18 \pm 0.14, p=0.189$ ). These free trial inactivation results provide a clear positive control for our PPC inactivations, demonstrating that the lack of effect on choice trials was not caused by a technical issue (like clogged cannula).

\section{A three-agent mixture model of risky choice}

While the effects of FOF silencing confirmed its role in decisions under risk (FIGURE $2 \mathrm{~B})$, the GLMM results did not provide insight into the specific role that the FOF might play. To better understand animal behavior in the task and the role of the FOF, we developed a three-agent mixture model (FIGURE 4A). The first agent is a 'rational', utility-maximizing agent (Von Neumann and Morgenstern, 1953) with two parameters: $\rho$, which controls the shape of the utility function $\left(U=V^{\rho}\right) ; \sigma$, which captures the decision noise. The other two agents were stimulus-independent agents which either habitually chose the lottery or the surebet. The relative influence of the agents is controlled by their mixing weights $\omega$, where $\sum \vec{\omega}=1$. The choice on each trial is thus a weighted outcome of the 'votes' of three agents, each implementing a different strategy. We estimated the joint posterior over the parameters for each subject separately using Hamiltonian Monte Carlo sampling in Stan (Carpenter et al., 2016) and validated that the model can correctly recover generative parameters from synthetic data (FIGURE S8A). Details of the modeling, including the priors, can be found in the Methods section. The motivation for developing the mixture model was that the animals' choices, while clearly sensitive to the lottery offer, showed some stimulus-independent biases. In other words, even for the best lottery they sometimes chose the surebet and for the worst lottery (which had a value of 0 ) they sometimes chose the lottery. For example, subject 2156 has a psychometric curve that asymptotes in a way that is inconsistent with a pure utility-maximizing strategy (FIGURE 4B).

Trial history effects could have been incorporated by allowing model parameters to vary depending on the outcome of the previous trial (as in Constantinople et al., $2019 b)$. However, our animals seemed to understand that the lottery offer was 
A
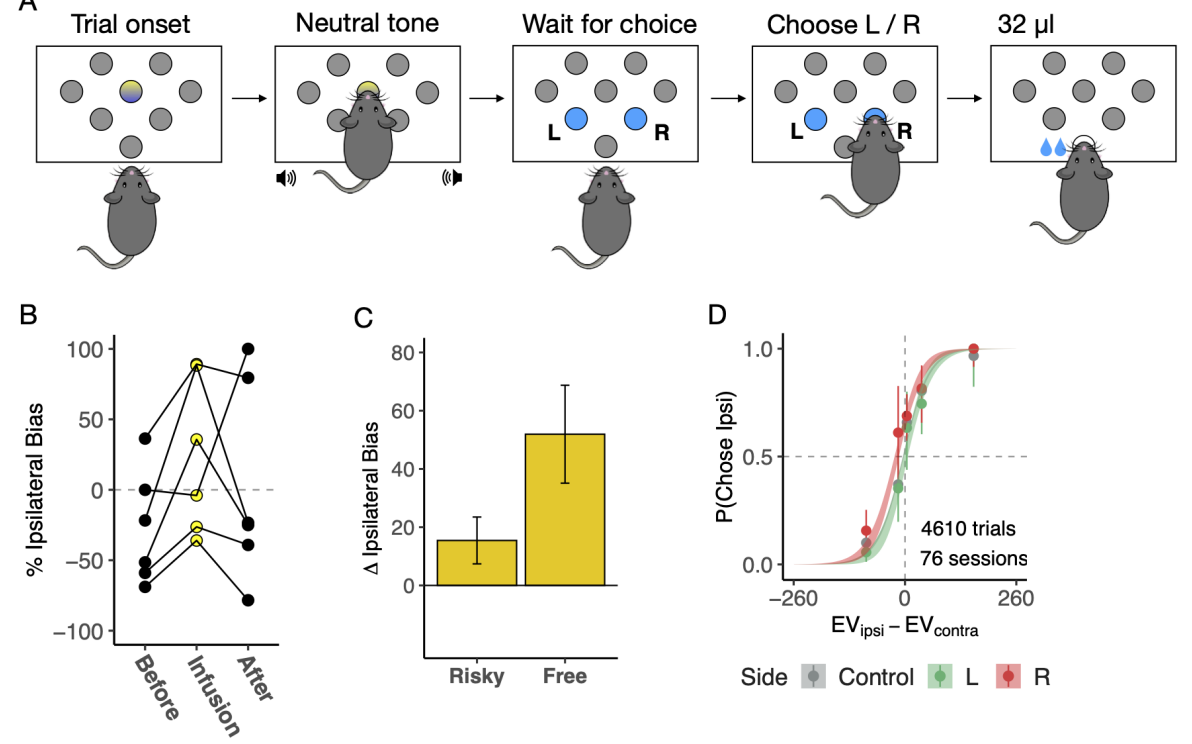

C

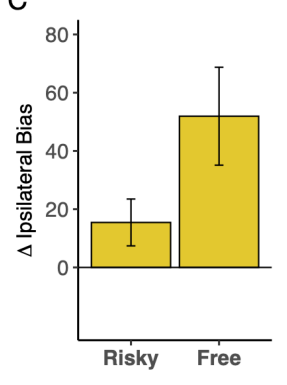

D

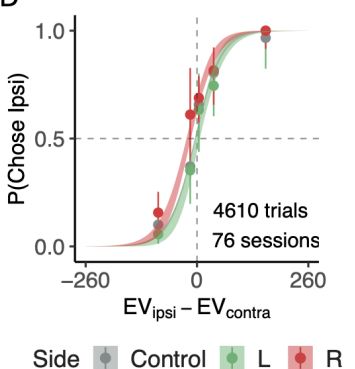

Figure 3. Unilateral PPC inactivation induced an ipsilateral bias on free trials. A. Schematic of the free trials. After fixation at the center port accompanied by a neutral tone, the animal was free to choose the left or right port, both illuminated in blue LEDs. Choosing either port resulted in a reward twice the magnitude of surebet. The free trials were randomly interleaved with the forced and choice trials. B. Unilateral PPC infusions $(0.6 \mu \mathrm{g})$ led to a significant ipsilateral bias towards the side of infusion. This panel shows \% ipsilateral bias: ( $\sum$ choose_infusion_side $-\sum$ choose_other_side) $/ \sum$ total_choices, when the side of infusions was chosen to be the opposite to the animals' preferred side. \% ipsilateral bias was computed using free trials from the previous 3 sessions, the infusion session, and the following 3 sessions for 6 subjects. C. Unilateral PPC infusions generated a significant $52 \pm 16 \%$ (mean \pm s.e. across rats, $\mathrm{n}=6$ ) change in $\%$ ipsilateral bias on free trials compared to control sessions (3 preinfusion sessions). For the choice trials from the same sessions, the change in $\%$ ipsilateral bias was not significant $(15 \pm 8 \%)$. D. Performance on the choice trials was not affected. Control sessions from the 3 pre-infusion sessions $(\mathrm{n}=65$ sessions, 6 rats $)$ are in gray, $0.6 \mu \mathrm{g}$ left PPC infusions ( $\mathrm{n}=5$ sessions) are in green, $0.6 \mu \mathrm{g}$ right $\mathrm{PPC}$ infusions $(\mathrm{n}=6$ sessions) are in red. 
independent across trials, and we did not see any significant effects of previous trial's outcome on choice in control sessions $\left(\beta_{\text {lottery }- \text { win }}=0.20 \pm 0.12, p=0.08\right.$;

$\left.\beta_{\text {lottery-lose }}=0.17 \pm 0.09, p=0.08\right)$. For this reason, we decided to formulate the three-agent mixture model without trial history parameters. Our animals' behavior stands in contrast to a substantial number of published results demonstrating strong trial history effects in rodent decision-making even when the optimal strategy is to only use information on the current trial (e.g. Constantinople et al., 2019b, Morcos and Harvey, 2016, Scott et al., 2015). We speculate that an important difference is that in traditional rodent two-alternative forced-choice tasks, the rewards were delivered at the choice ports, but in our task all rewards were delivered at a single reward port (but for counter examples where there is history dependence despite using a single reward port, see The International Brain Laboratory et al., 2021, Zalocusky et al., 2016).

The three-agent model fit the control behavior well (see two example animals in Figure 4B, all animals in Figure S8B). For example, rat 2156 chooses the lottery about $10 \%$ of the time for the 4 worst lotteries. This behavior is not well described by a 'pure' utility-maximizing model. All animals had a decelerating utility function (95\% C.I. of $\rho<1$ for all animals; FIguRE 4C). Note that the effective risk-preference is influenced by both $\rho$ and $\omega$. For example, the indifference point of 2152 is close to 0 , implying that it is effectively risk-neutral (FIGURE 4B). However, this comes from its bias towards choosing the lottery $\left(\omega_{\text {lottery }}=0.16\right)$ balancing its decelerating utility function $(\rho=0.62$; Table 1$)$. The animals had small but varying levels of decision noise $(\sigma=1.00$ [0.35 3.40], median and 95\% C.I. of concatenated posteriors across animals), indicating that they were sensitive to water rewards just a few $\mu \mathrm{L}$ apart. Their choices were guided mostly by the rational agent $\left(\omega_{\text {rational }}=0.82[0.650 .95]\right)$, with little influence from the lottery agent $\left(\omega_{\text {lottery }}=0.10[0.010 .31]\right)$ and the surebet agent $\left(\omega_{\text {surebet }}=0.05[0.010 .23]\right)$.

\section{Bilateral FOF inactivation reduced the utility exponent $\rho$}

In order to quantify how the infusions influenced model parameters, we constructed a new version of the three-agent model that fit the $0.3 \mu \mathrm{g}$ bilateral FOF and $0.3 \mu \mathrm{g}$ bilateral PPC infusion data as perturbations of the control dataset for each subject (Figure 5A \& Figure S11). We chose priors for the effects of perturbation such that the model favored no effect of inactivation (i.e. zero mean for shifts and one mean for scaling effects). Bilateral PPC infusion led to no reliable changes across subjects for all parameters, which was consistent with the results from the GLMM (FIGURE S11 \& TABLE 1). From the GLMM (and visual inspection), we knew that bilateral FOF silencing substantially shifted the subjects to being effectively more risk-averse. Indeed, the model-based analysis showed that almost all animals had a reduction in $\rho$ compared to the control fits (FIGURE 5B, first column). In contrast, other parameters did not show a consistent direction of change across animals (FIGURE 5B).

To validate that the reduction in lottery choices was actually due to a decreased $\rho$ rather than an increased $\omega_{\text {surebet }}$, we constructed two variants of the inactivation model and compared them using 10-fold cross validation (see Methods for details). The ' $\rho$-only' model had parameters allowing $\rho$ and $\sigma$ to shift, but not any $\omega$ parameters to change under inactivation. Similarly, the ' $\omega$-only' model had parameters allowing only $\omega$ and $\sigma$ but not $\rho$ to change. The ' $\rho$ and $\omega$ ' model was the standard inactivation model that allowed every parameter to change under inactivation. We found that in 6 out of 8 subjects, model comparison result preferred the $\rho$-only model over the $\omega$-only model (FIGURE $5 \mathrm{C}$ ). The $\omega$-only model was strongly preferred only in one subject's dataset (2152). Taken together, these results suggest that the most parsimonious interpretation of the inactivation-induced effect is a reduction in the utility exponent, $\rho$. 
A

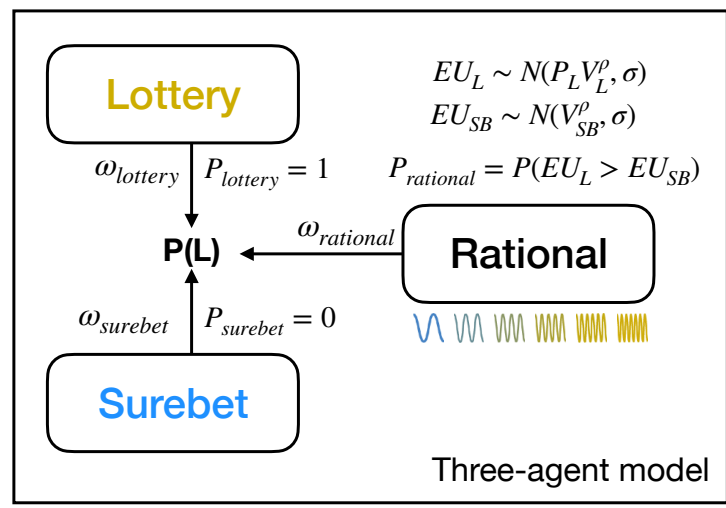

C

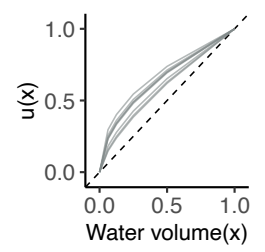

D

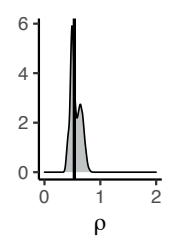

B
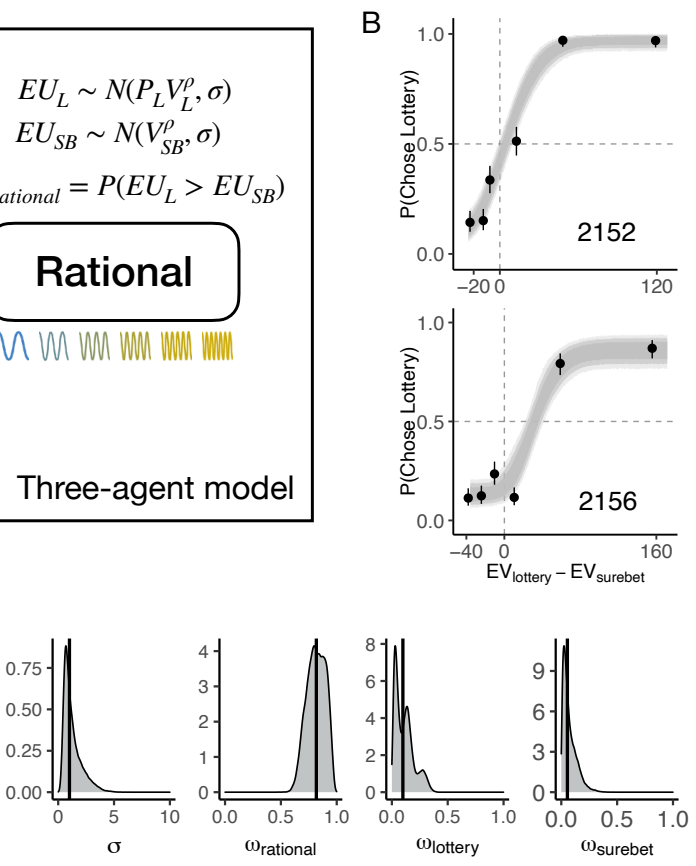

Figure 4. The three-agent mixture model and model fits. A. The three-agent mixture model. The animal's choice is modelled as a weighted average of the three agents, each implementing a different behavioral strategy to perform the task. Each agent outputs a probability of choosing lottery that makes up the probability vector $\vec{P}$, which is combined using their respective weights $\vec{\omega}$. See Methods for model details. B. The three-agent mixture model can fit the control behavior well. The circles with error bars are the binned mean and $95 \%$ binomial confidence intervals. The ribbons are model predictions generated using the fitted parameters. The dark, medium and light shade represent $80 \%, 95 \%$ and $99 \%$ confidence intervals, respectively. C. The subjective utility functions for each rat computed using max a posteriori $\rho$ estimation, normalized by the maximum water volume. D. Density plots of concatenated posterior samples (4000 each) from the model fits across 8 animals. The black bar is the median of the distribution. 
How can silencing the FOF change the exponent of the utility function? Previous silencing and modeling results suggested that the FOF is part (1/6) of a distributed circuit for maintaining a prospective memory of choice (Kopec et al., 2015). Inspired by that finding, we constructed a 6 -node rate model of a distributed circuit for encoding action-value, where the FOF represented one node in that network (FIGURE 6A; Burak and Fiete, 2012). Three nodes other than the FOF node received input representing the magnitude of the lottery. The all-to-all weight matrix was generated randomly, but the distribution of the weights was chosen such that the response of the network to the inputs was in the dynamic regime of the nodes $(0<H z<100)$. Other network parameters (noise $\sigma$ and time-constant $\tau$ ) were chosen to generate a control network response with reasonable dynamics (FIGURE 6B) that encoded the lottery value in the population activity of the network (FIGURE 6C, gray circles). In this regime, we found that silencing the FOF node scaled down the network's responses. We can think of this network as encoding the expected utility of choosing the lottery by transforming the lottery sound into utils (encoded as spike rate). At the time of the go-cue, this activity could become bistable: where the utility of the surebet determines the unstable fixed point (similar to Machens, 2005). Alternatively, a downstream region could compare the output of this network with the remembered surebet utility. In any case, scaling down the input-output transform of the network (FIGURE 6C, purple circles) would shift the indifference point (the lottery that had the same activity level as the surebet comparator), which would, behaviorally, appear as a change in the power-law utility function $U=V^{\rho}$. For the control network, the network approximates a function with $\rho \approx 0.76$. After silencing the FOF node, the exponent of the utility functions shifted down, $\rho \approx 0.6$ (FIgURE $6 \mathrm{C}$ ). This biophysical model provides a mechanistic explanation for our finding that silencing the FOF with muscimol caused animals to avoid choosing the lottery (FIGURE 2B) through a change in the exponent of the utility function (FIGURE 5B).

\section{Bilateral PPC inactivation did not impair learning}

The GLMM analysis above shows that the rat PPC was not causally involved in the risky choice task. However, numerous studies have found that neural activity in PPC correlated with decision variables in both perceptual and economic tasks. The question thus remains, what is the purpose of these decision-related signals in PPC? Recently, Zhong et al. (2019) found that PPC silencing impaired the ability of mice to re-categorize previously experienced stimuli based on a new category boundary in an auditory decision-making task. Moreover, after the stimuli were re-categorized, PPC activity was no longer required for performance. Motivated by their findings, we tested whether PPC was necessary for re-categorizing stimuli in our task. To do so, we employed a model-based change in the surebet magnitude that effectively shifted the decision boundary without changing the frequency-to-lottery mapping (FIGURE 7A). As such, some frequencies that were previously preferred over the surebet became unpreferred (and vice-versa, depending on the direction of the shift). To estimate the required shifts, we first fit the three-agent model on data from the past 14 sessions. We then used the fit to generate synthetic choices on different surebet magnitudes, until we found the one that resulted in a shift in the overall probability of choosing lottery ( $p$ (Choose Lottery)) close to the target (drawn uniformly from $\pm U(0.2,0.3)$; see details in Methods). To familiarize animals with the new paradigm, their surebet magnitudes were changed weekly for two weeks prior to any infusion. Two out of six animals failed to show appropriate adaptation of behavior following change in surebet magnitude; they were excluded from analysis in this section. The other four animals reliably shifted their choices more towards surebet when its magnitude increased, and more towards lottery when its magnitude decreased (See example animal in FiguRE 
A
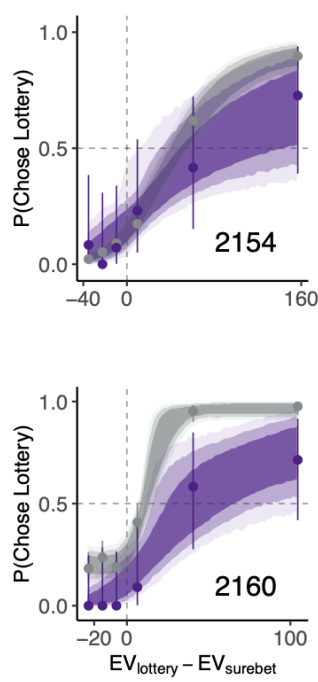

B

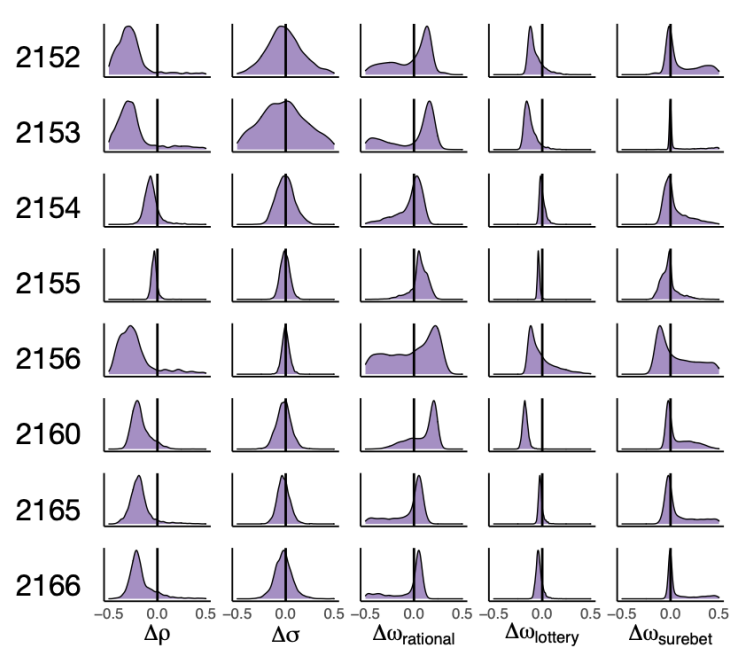

C

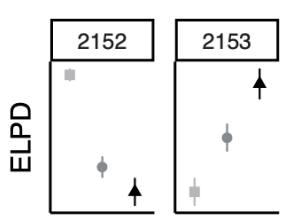

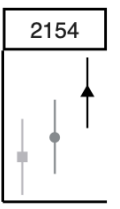
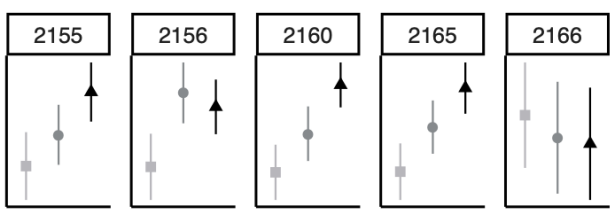

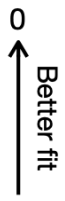

+ $\omega$-only $\phi \rho$ and $\omega$ \& $\rho$-only

Figure 5. Bilateral FOF inactivation reduced the utility exponent. A. Psychometric curves for two example animals. The circles with error bars are the binned mean and $95 \%$ binomial confidence intervals. The ribbons are model predictions generated using the fitted parameters (control-gray, bilateral FOF inactivation-purple). The dark, medium and light shade represent $80 \%, 95 \%$ and $99 \%$ confidence intervals, respectively. All animals are shown in Figure S10. B. Normalized (with peak at 1) density plots showing deviation of the model parameters under bilateral FOF inactivation from control. The most reliable finding across animals is that $\Delta \rho$ is negative. C. Ten-fold cross-validation results comparing three model variants: where $\omega$ but not $\rho$ was allowed to change under the inactivation dataset, both $\rho$ and $\omega$ were allowed to change, and $\rho$ but not $\omega$ was allowed to change. The points with error bars are the expected $\log$ posterior density (ELPD) and its standard error on each animal's dataset. The $\rho$-only model was preferred to the $\omega$-only model in 6 out of 8 animals. 

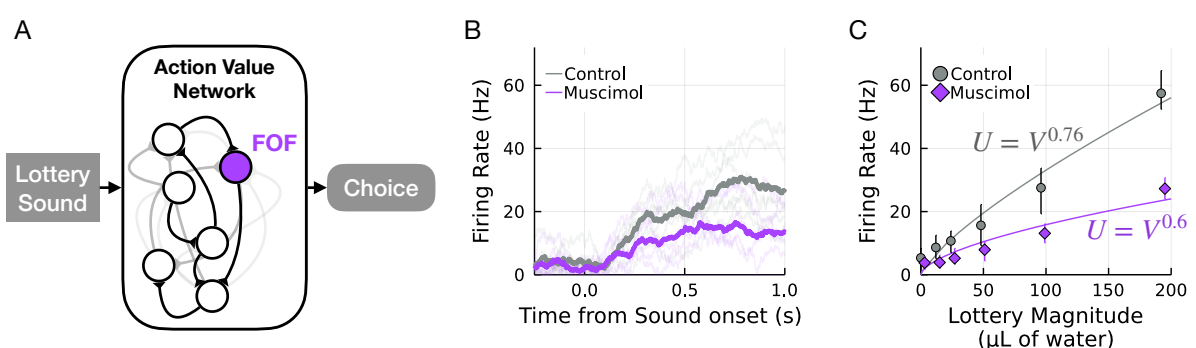

Figure 6. Biophysical model of FOF silencing. A. We implemented a 6-node rate model of a distributed action-value network with random connectivity $\left(W_{i j} \sim \mathcal{N}(5 / 6,1)\right)$. The FOF is 1 of the 6 nodes (in purple). The input to the network was the lottery magnitude. For the following plots the random seed for generating $W$ was set to 131 and then that $W$ was used for all further simulation, but similar results can be obtained with other $W$ generated with the same statistics from a different seed. B. Example of the network response to lottery sound with magnitude of $96 \mu \mathrm{L}$ under control conditions (with all the nodes active, in grey) and under FOF silencing (the FOF node is set to zero, in purple). The dark traces represent the mean network activity and the light traces represent the activity of the 6 individual nodes. C. Silencing FOF scales down the representation of the action-value of the lottery, which could explain the shift in $\rho$. We ran the network for 20 'trials' of each lottery $\in[0,12,24,48,96,192] \mu \mathrm{L}$. The grey circle are the mean and $95 \%$ CI for the network response in the control conditions and the purple diamonds are the mean and $95 \%$ CI for the network response when the FOF node is silenced. Fitting a power-law utility function, $U=V^{\rho}$ to the network activity gives $\rho \approx 0.76$ for control, and after FOF silencing $\rho \approx 0.6$. The thin lines are power-law utility functions that approximate the transformation from units of reward $(\mu \mathrm{L})$ to utils in spikes / second.

7B, all other animals in Figure S12)

After two weeks, on the day of surebet change, we infused $0.6 \mu \mathrm{g}$ muscimol into each side of PPC in these four animals. The animals learned the new surebet magnitude and adjusted their behavior to the same extent in control and PPC inactivation sessions (see example animal in Figure 7C). To validate that the animals adapted in a preference-preserving manner, we fit one model to all the sessions with different surebet magnitudes for each animal. We then used these parameters to predict the shift in $p$ (Choose Lottery) on the day of change, and compared it against the actual shift in $p$ (Choose Lottery) in that session (Figure 7D). The model was able to accurately predict the shift $(R=0.905, p<0.001)$, suggesting that the animals adapted their choices without altering the underlying preference and strategy. Bilateral PPC inactivation did not impair the learning of new surebet magnitudes, as the shifts in $p$ (Choose Lottery) on the day of change were not statistically different between the infusion and control sessions $\left(T_{8.7}=0.44, p=0.67\right)$. Note that the surebet learning experiment was done before the free trial control experiment, so the lack of effect cannot be dismissed as a technical issue (i.e. infusions in the PPC were not effective at silencing activity). Overall, our results do not support the hypothesis that the PPC is required for shifting category boundaries: i.e. categorizing a lottery as being better or worse than the surebet. 
A

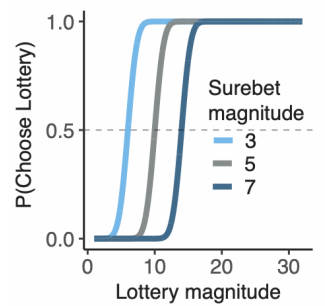

B

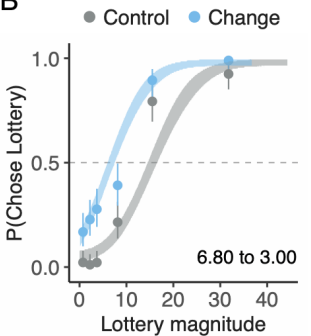

C

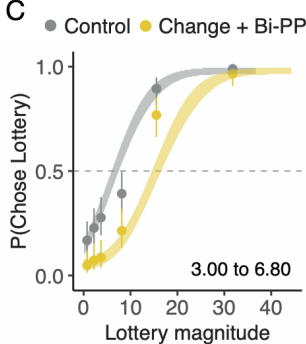

D C Change

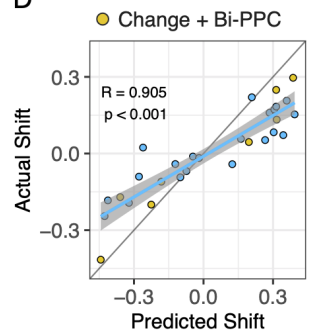

Figure 7. Bilateral PPC inactivations did not impair learning of new surebet magnitudes. A. Schematic showing changing the surebet magnitude is equivalent to shifting the choice boundary. The data points were simulated from a risk-neutral agent using the three-agent model $\left(\rho=1, \sigma=3, \omega_{\text {rational }}=1\right)$. A smaller surebet magnitude (light blue) horizontally shifts the psychometric curve leftwards, a larger surebet magnitude (dark blue) shifts the curve rightwards. The frequency-to-lottery mapping remains the same. B. Changing surebet magnitude from 6.8 to 3 shifted choices leftwards in one example animal. Combined trials from 6 sessions before the change are shown in gray, after the change shown in blue. One threeagent model was fit to all the trials and the parameters were used for ribbon extrapolation. C. Same as B but with $0.6 \mu \mathrm{g}$ per side bilateral PPC infusion, performed on the day of surebet change (from 3 to 6.8). D. The three-agent mixture model predicts the shifts in behavior well. One model was fit using all the sessions containing various surebet magnitudes for each animal. On x-axis is the predicted shift in probability choosing lottery ( $p$ (Choose Lottery)): the difference in $p$ (Choose Lottery) between model prediction using the new surebet magnitude and the session just before that change. On y-axis is the actual shift in $p$ (Choose Lottery): the difference in $p$ (Choose Lottery) between the first session of a surebet change and the session before that change. Sessions with just surebet change are in blue ( $\mathrm{n}=21 ; 4$ animals), sessions with both surebet change and $0.6 \mu \mathrm{g}$ per side bilateral PPC infusions are in gold $(\mathrm{n}=8)$. The model prediction strongly correlated with the actual shift $(R=0.905, p<0.001)$. No significant difference in the actual shift was found between the blue and gold sessions $\left(T_{8.7}=\right.$ $0.44, p=0.67)$. 


\begin{tabular}{|c|c|c|c|c|c|c|}
\hline & & $\rho$ & $\sigma$ & $\omega_{\text {rational }}$ & $\omega_{l o t t e r y}$ & $\omega_{\text {surebet }}$ \\
\hline \multirow{3}{*}{2152} & Control & $0.62[0.59,2.07]$ & $1.61[1.16,3.06]$ & $0.82[0.64,0.87]$ & $0.16[0.11,0.18]$ & $0.02[0.01,0.20$ \\
\hline & FOF & $0.38[0.11,9.07]$ & $1.54[1.10,3.21]$ & $0.97[0.14,1.00]$ & $0.02[0.00,0.28]$ & $0.00[0.00,0.70$ \\
\hline & PPC & $0.66[0.58,0.78]$ & $1.62[1.12,3.12]$ & $0.78[0.64,0.87]$ & $0.14[0.06,0.21]$ & $0.09[0.04,0.20$ \\
\hline \multirow{3}{*}{2153} & Control & $0.67[0.65,2.41]$ & $2.52[1.39,4.63]$ & $0.79[0.62,0.86]$ & $0.20[0.14,0.23]$ & $0.00[0.00,0.18$ \\
\hline & FOF & $0.42[0.13,6.71]$ & $2.32[1.38,4.77]$ & $0.97[0.03,1.00]$ & $0.02[0.00,0.22]$ & $0.00[0.00,0.90$ \\
\hline & PPC & $0.62[0.56,0.71]$ & $2.37[1.36,4.79]$ & $0.89[0.80,0.99]$ & $0.09[0.01,0.16]$ & $0.02[0.00,0.06$ \\
\hline \multirow{3}{*}{2154} & Control & $0.45[0.42,0.50]$ & $1.05[0.73,1.21]$ & $0.96[0.82,0.98]$ & $0.02[0.01,0.06]$ & $0.03[0.00,0.14$ \\
\hline & $\mathrm{FOF}$ & $0.35[0.28,0.65]$ & $0.84[0.69,1.27]$ & $0.99[0.46,1.00]$ & $0.01[0.00,0.12]$ & $0.00[0.00,0.48$ \\
\hline & $\mathrm{PPC}$ & $0.43[0.39,0.52]$ & $0.90[0.68,1.27]$ & $0.93[0.72,0.97]$ & $0.07[0.02,0.15]$ & $0.00[0.00,0.19$ \\
\hline \multirow{3}{*}{2155} & Control & $0.39[0.36,0.42]$ & $0.48[0.43,0.65]$ & $0.84[0.77,0.96]$ & $0.05[0.03,0.07]$ & $0.10[0.00,0.18$ \\
\hline & FOF & $0.33[0.31,0.42]$ & $0.49[0.41,0.66]$ & $0.96[0.68,1.00]$ & $0.02[0.00,0.04]$ & $0.01[0.00,0.31$ \\
\hline & PPC & no data & no data & no data & no data & no data \\
\hline \multirow{3}{*}{2156} & Control & $0.55[0.46,0.59]$ & $0.19[0.22,0.56]$ & $0.71[0.66,0.76]$ & $0.14[0.12,0.17]$ & $0.15[0.10,0.19$ \\
\hline & $\mathrm{FOF}$ & $0.22[0.05,2.34]$ & $0.15[0.21,0.58]$ & $0.56[0.05,0.99]$ & $0.29[0.00,0.51]$ & $0.15[0.00,0.81$ \\
\hline & PPC & $0.50[0.42,0.66]$ & $0.21[0.22,0.59]$ & $0.61[0.56,0.86]$ & $0.09[0.03,0.12]$ & $0.30[0.08,0.36$ \\
\hline \multirow{3}{*}{2160} & Control & $0.66[0.56,0.67]$ & $0.59[0.48,1.17]$ & $0.76[0.72,0.82]$ & $0.21[0.16,0.24]$ & $0.03[0.01,0.06$ \\
\hline & FOF & $0.32[0.33,0.66]$ & $0.58[0.47,1.15]$ & $0.98[0.53,1.00]$ & $0.02[0.00,0.07]$ & $0.01[0.00,0.43$ \\
\hline & $\mathrm{PPC}$ & $0.54[0.50,0.70]$ & $0.50[0.46,1.22]$ & $0.82[0.75,0.96]$ & $0.17[0.03,0.18]$ & $0.01[0.00,0.12$ \\
\hline \multirow{3}{*}{2165} & Control & $0.45[0.43,0.50]$ & $0.34[0.41,0.92]$ & $0.88[0.84,0.97]$ & $0.07[0.01,0.06]$ & $0.06[0.01,0.12$ \\
\hline & $\mathrm{FOF}$ & $0.29[0.11,0.80]$ & $0.37[0.38,0.94]$ & $0.99[0.10,1.00]$ & $0.01[0.00,0.08]$ & $0.00[0.00,0.87$ \\
\hline & $\mathrm{PPC}$ & $0.43[0.37,0.53]$ & $0.29[0.41,0.96]$ & $0.98[0.82,1.00]$ & $0.01[0.00,0.06]$ & $0.01[0.00,0.15$ \\
\hline \multirow{3}{*}{2166} & Control & $0.43[0.43,0.54]$ & $0.61[0.51,1.51]$ & $0.92[0.87,0.97]$ & $0.07[0.02,0.10]$ & $0.01[0.00,0.05$ \\
\hline & FOF & $0.22[0.14,1.01]$ & $0.49[0.47,1.55]$ & $1.00[0.21,1.00]$ & $0.00[0.00,0.10]$ & $0.00[0.00,0.75$ \\
\hline & $\mathrm{PPC}$ & $0.57[0.28,0.88]$ & $0.68[0.50,1.58]$ & $0.82[0.51,0.99]$ & $0.03[0.01,0.37]$ & $0.15[0.00,0.23$ \\
\hline
\end{tabular}

Table 1. Fits from the three-agent inactivation model. Statistics were computed using the parameter posteriors from the three-agent model fit to the control, $0.3 \mu \mathrm{g}$ per side bilateral FOF inactivation, and $0.3 \mu \mathrm{g}$ per side bilateral PPC inactivation dataset simultaneously. The median of the parameter posterior distribution is reported along with its $95 \%$ confidence interval in brackets. 


\section{Discussion}

The neurobiology of decision-making under risk has been studied extensively in humans, non-human primates and rodents. However, there has been a gap in task design between the human-primate and rodent experiments, that most rodent studies focused on unexpected uncertainty where choices often reflected their sensitivity of reward history rather than risk attitudes. Here, we developed a risky choice task for rats, where animals made cue-guided decisions between a lottery and a surebet option on a trial-by-trial basis under expected uncertainty, as in human and primate experiments (but see Constantinople et al., 2019a, Hocker et al., 2021, for recent examples of rodent work on expected uncertainty). We developed the three-agent mixture model to decompose different elements of risk-preference, including $\rho$ as exponent on the utility curve and $\vec{\omega}$ as the weights for the rational, lottery and surebet agents. Modeling results showed that all subjects had decelerating, i.e. risk-averse, utility functions and their decisions were influenced mostly by the rational agent. We tested how inactivations of two cortical regions, the FOF and PPC, influenced choices in our task. These regions have been studied extensively in perceptual decision-making, but this is the first test of their casual role in economic decision-making. Bilateral FOF inactivations produced a profound bias towards the surebet, while bilateral PPC inactivations had minimal effect on the behavior.

Model-based analyses of the results indicated that, without the FOF, subjects had utility functions that were substantially shifted towards risk-aversion. We constructed a biophysical model to show that inactivating the FOF node can produce outputs similar to the effects observed, providing a mechanistic explanation. Finally, we found that PPC was not causally involved in the learning of new categorization boundaries.

\section{Role of FOF}

Results from bilateral FOF inactivations show that FOF is an essential part of the circuitry underlying risky decision-making. Model-based analyses suggest that the change in behavior was likely due to a decrease in $\rho$, the curvature of the utility function, $U=V^{\rho}$. However, due to the small number of trials collected in bilateral FOF inactivation sessions, we cannot say definitively that the effect was exclusively on $\rho$; it may have been a combination of both decreasing $\rho$ and also increasing influence from the surebet agent on choice. Additionally, there are many functional forms of decision-making under uncertainty that we did not test (Chen and Stuphorn, 2018, Farashahi et al., 2019, Weber et al., 2004), which could lead to different interpretations. Nevertheless, the change in $\rho$ is consistent with the finding that inactivation of monkey supplemental eye field reduced risky choices and the change was characterized by a decreased utility exponent (Chen and Stuphorn, 2018). Using a dynamical model, we demonstrated that a shift in $\rho$ can be caused by a partial inactivation of an action-value network whose activity guides choice (Samejima et al., 2005). This is similar to the theory that the FOF is part of a network for planning upcoming choice (Kopec et al., 2015). In fact, the interpretation of FOF activity encoding action-value is consistent with the previous interpretation (planning movement), since in perceptual tasks, only the correct side is rewarded, making it difficult to disentangle action-value from movement-planning. This action-value network may be the locus of transformation from value to utility, with the network properties (e.g. whether the gain of the network is greater or less than 1) determining whether animals are risk-seeking or risk-averse. One key difference between our current and previous findings, is that previously, sensory-guided choices (i.e. trials with no working-memory requirement) were not affected by silencing FOF (Erlich et al., 2015, Kopec et al., 2015). In Erlich et al. (2015), we posited that the FOF may 
be a bottleneck through which long-timescale integration of information could influence orienting decisions. Our results here suggest that the FOF may play a similar role for decisions that require integration of multiple attributes - in this case, lottery value and probability.

The idea that the FOF is part of an action-value network is largely consistent with the view that the FOF contributes to sensoryto-to-motor transformation (Barthas and Kwan, 2017, Ebbesen et al., 2018, Insanally et al., 2019, Scott et al., 2017, Siniscalchi et al., 2016), but reinterprets those findings as sensory-to-value transformations. Whether the same network also directly guides movement or transmits action-values to a downstream action-selection circuit (Essig et al., 2021, Shires et al., 2010) is an interesting question for future work: it has been previously demonstrated that changes in, e.g. excitatory drive, driven by a go-cue, can shift a network from monotonically encoding a task variable to encoding a binary choice (Machens, 2005). It is important to note that, in our task, the surebet value is stable across trials and only the lottery needs to be evaluated on a trial-by-trial basis. We predict that in a task where the lottery is stable across trials and the surebet value varies trial-by-trial (and is indicated by a cue), silencing the FOF would shift animals away from selecting the surebet, whose value would require transformation on each trial. In tasks that require transformations for both surebet and lottery (e.g. Constantinople et al., 2019a), bilateral FOF silencing might result in increased decision noise.

Both left and right unilateral FOF inactivations led to a small bias towards leftward choices (FIGURE 2C), rather than an contralateral impairment, as previously reported (Erlich et al., 2015, Hanks et al., 2015, Kopec et al., 2015). However, in those studies, trials that did not require short-term memory were not biased by unilateral FOF inactivations, so the small effect is not particularly surprising. As 7 out of 8 animals had the surebet port on the left, the leftward bias can be interpreted as a weak bias towards choosing the surebet; i.e. a partial effect consistent with our bilateral silencing results.

\section{Role of PPC in risky choice}

Activity in PPC has long been be associated with decision variables in economic choices. Platt and Glimcher (1999) first showed that activity in monkey lateral intraparietal cortex (LIP), a visuomotor area within PPC, is sensitive to expected reward magnitude and probability. Subsequently, Dorris and Glimcher (2004) found that neurons in monkey LIP encode relative subjective desirability of actions in a mixed-strategy game. Activity in human PPC also correlates with subjects' risk preferences (Huettel et al., 2006). To date, we are not aware of any studies of the role of rodent PPC in economic decisions. Nonetheless, PPC encodes task-related variables during perceptual decisions (e.g. Goard et al., 2016, Hanks et al., 2015, Raposo et al., 2014). We were frankly disappointed that neither unilateral nor bilateral inactivation had any effect on the risky choices. As far as we are aware, this is the first experiment that directly tests the causal role of PPC in economic choices.

The null result is reminiscent of the null effects of PPC inactivation in the Poisson clicks task in rats (Erlich et al., 2015), and of LIP inactivation in the random dot task in monkeys (Katz et al., 2016). The null effect was unlikely the result of insufficient inactivation, as unilateral PPC infusions led to a significant ipsilateral bias in the free choice trials, where the decisions were guided by internal side preference rather than action value (FIGURE 3A). The free choice result replicates previous findings (Erlich et al., 2015, Katz et al., 2016), and is consistent with the literature on the role of rodent PPC in neglect (Bucci, 2009, Reep and Corwin, 2009), providing a clear positive control for the inactivation experiments. Taken together, our results demonstrate that PPC is not strictly necessary for making utility maximizing choices 
under risk.

It has been argued that rodent PPC is important for visually-guided decisions but not other modalities. For example, pharmacological inactivation of PPC has been shown to impair mice' performance in a visually-guided navigation task with a memory component (Harvey et al., 2012), and in a multi-sensory perceptual task but when only using visual but not auditory cues (Raposo et al., 2014). It was suspected that due to the anatomical proximity between PPC and the visual areas, these inactivation results may be caused by a muscimol spillover into the adjacent visual cortex. However, recent experiments utilizing optogenetics have shown that, targeted inactivation of PPC during the stimulus period disrupted performance only on the visual but not auditory trials (Licata et al., 2017), and impaired decision sensitivity in a visually-guided task with variable delays in mice (Goard et al., 2016). As such, we cannot exclude the possibility that the null effect on risky choice may be due to the modality of stimuli used. However, silencing mouse PPC was shown to impair re-categorization of sounds in an auditory task (Zhong et al., 2019), so the controversy over the modality-specific role of PPC is not fully resolved.

\section{Role of PPC in learning}

We have shown that inactivating PPC did not impair the animal's ability to shift their choices in response to changes in the value of the surebet, inconsistent with the findings from Zhong et al. (2019). There are some key differences in the design between our and their experiments that may explain this. First, their experiments were performed on head-fixed mice, whereas our rats were freely moving in the training box. Second, their mice had to categorize (or re-categorize) stimuli for the first time while PPC was inactivated. They never tested whether PPC was required for learning recurring shifts in the decision boundary. In contrast, the animals in our experiment were accustomed to changing surebet values for two weeks prior to PPC inactivation, understanding that the surebet value may change unexpectedly. Bucci and Chess (2005) found that PPC-lesioned rats had trouble learning the association between light and food if previously the light was presented without food. Interestingly, normal learning was observed in another cohort of PPC-lesioned rats that were not pre-exposed to the light. They attributed the impairment to PPC's role in directing attention to the stimulus whose meaning surprisingly changes. If it is the case that PPC activity is required for the learning of 'surprising' shifts in existing associations, the discrepancy between our experiment and Zhong et al.'s can be then resolved.

\section{Conclusion}

Studies of neurobiology of economic choice in rodents have mostly focused on the reward-valuation circuit: including the amygdala (Larkin et al., 2016, Orsini et al., 2017), basal ganglia (Stopper et al., 2013) and orbital-frontal cortex (Constantinople et al., 2019a, Gardner et al., 2017, Hocker et al., 2021, Kuwabara et al., 2020, Ogawa et al., 2013, Roesch et al., 2006). Here, we examined the casual contribution of two cortical areas associated with planning orienting decisions, the FOF and PPC, whose analogous primate regions have been implicated in economic decision-making (Chen and Stuphorn, 2018, Platt and Glimcher, 1999). We found that FOF is a critical node in the circuit for decisions under risk, while PPC is not. Our results predict that FOF neurons participate in sensory-to-value transformation and would increase their activity monotonically with action-value. 


\section{Acknowledgments}

We thank Yidi Chen, Anyu Fang, NengNeng Gao, Yingkun Li and Cequn Wang for technical assistance related to building and maintaining lab infrastructure as well as training animals and assisting with infusions. JCE acknowledges the support of the 111 project (Base B16018), the National Natural Science Foundation of China (NSFC) and the support of the NYU-ECNU Institute of Brain and Cognitive Science at NYU Shanghai.

\section{References}

23and Me Research Team, eQTLgen Consortium, International Cannabis Consortium, Social Science Genetic Association Consortium, R. Karlsson Linnér, P. Biroli, E. Kong, S. F. W. Meddens, R. Wedow, M. A. Fontana, M. Lebreton, S. P. Tino, A. Abdellaoui, A. R. Hammerschlag, M. G. Nivard, A. Okbay, C. A. Rietveld, P. N. Timshel, M. Trzaskowski, R. de Vlaming, C. L. Zünd, Y. Bao, L. Buzdugan, A. H. Caplin, C.-Y. Chen, P. Eibich, P. Fontanillas, J. R. Gonzalez, P. K. Joshi, V. Karhunen, A. Kleinman, R. Z. Levin, C. M. Lill, G. A. Meddens, G. Muntané, S. Sanchez-Roige, F. J. van Rooij, E. Taskesen, Y. Wu, F. Zhang, A. Auton, J. D. Boardman, D. W. Clark, A. Conlin, C. C. Dolan, U. Fischbacher, P. J. F. Groenen, K. M. Harris, G. Hasler, A. Hofman, M. A. Ikram, S. Jain, R. Karlsson, R. C. Kessler, M. Kooyman, J. MacKillop, M. Männikkö, C. Morcillo-Suarez, M. B. McQueen, K. M. Schmidt, M. C. Smart, M. Sutter, A. R. Thurik, A. G. Uitterlinden, J. White, H. de Wit, J. Yang, L. Bertram, D. I. Boomsma, T. Esko, E. Fehr, D. A. Hinds, M. Johannesson, M. Kumari, D. Laibson, P. K. E. Magnusson, M. N. Meyer, A. Navarro, A. A. Palmer, T. H. Pers, D. Posthuma, D. Schunk, M. B. Stein, R. Svento, H. Tiemeier, P. R. H. J. Timmers, P. Turley, R. J. Ursano, G. G. Wagner, J. F. Wilson, J. Gratten, J. J. Lee, D. Cesarini, D. J. Benjamin, P. D. Koellinger, and J. P. Beauchamp. Genome-wide association analyses of risk tolerance and risky behaviors in over 1 million individuals identify hundreds of loci and shared genetic influences. Nature Genetics, 51(2):245-257, Feb. 2019. ISSN 1061-4036, 1546-1718. doi: 10.1038/s41588-018-0309-3.

S. H. Ahmed. Individual decision-making in the causal pathway to addiction: Contributions and limitations of rodent models. Pharmacology Biochemistry and Behavior, 164:22-31, Jan. 2018. ISSN 0091-3057. doi: 10.1016/j.pbb.2017.07.005.

A. P. Anokhin, S. Golosheykin, J. Grant, and A. C. Heath. Heritability of Risk-Taking in Adolescence: A Longitudinal Twin Study. Twin Research and Human Genetics, 12(4):366-371, Aug. 2009. ISSN 1832-4274, 1839-2628. doi: 10.1375/twin.12.4.366.

F. Barthas and A. C. Kwan. Secondary Motor Cortex: Where 'Sensory' Meets 'Motor' in the Rodent Frontal Cortex. Trends in Neurosciences, 40(3):181-193, Mar. 2017. ISSN 0166-2236. doi: 10.1016/j.tins.2016.11.006.

D. Bates, M. Mächler, B. Bolker, and S. Walker. Fitting Linear Mixed-Effects Models Using lme4. Journal of Statistical Software, 67(1):1-48, Oct. 2015. ISSN 1548-7660. doi: $10.18637 /$ jss.v067.i01.

T. E. J. Behrens, M. W. Woolrich, M. E. Walton, and M. F. S. Rushworth. Learning the value of information in an uncertain world. Nature Neuroscience, 10(9): 1214-1221, Sept. 2007. ISSN 1097-6256. doi: 10.1038/nn1954. 
J. Bezanson, A. Edelman, S. Karpinski, and V. B. Shah. Julia: A Fresh Approach to Numerical Computing. SIAM Review, 59(1):65-98, Jan. 2017. ISSN 0036-1445, 1095-7200. doi: 10.1137/141000671.

D. J. Bucci. Posterior parietal cortex: An interface between attention and learning? Neurobiology of Learning and Memory, 91(2):114-120, Feb. 2009. ISSN 1074-7427. doi: 10.1016/j.nlm.2008.07.004.

D. J. Bucci and A. C. Chess. Specific changes in conditioned responding following neurotoxic damage to the posterior parietal cortex. Behavioral Neuroscience, 119(6): 1580-1587, 2005. ISSN 1939-0084(Electronic),0735-7044(Print). doi: 10.1037/0735-7044.119.6.1580.

Y. Burak and I. R. Fiete. Fundamental limits on persistent activity in networks of noisy neurons. Proceedings of the National Academy of Sciences, 109(43): 17645-17650, Oct. 2012. ISSN 0027-8424, 1091-6490. doi: 10.1073/pnas.1117386109.

B. Carpenter, A. Gelman, M. Hoffman, D. Lee, B. Goodrich, M. Betancourt, M. A. Brubaker, J. Guo, P. Li, and A. Riddell. Stan: A probabilistic programming language. Journal of Statistical Software, 20:1-37, 2016.

X. Chen and V. Stuphorn. Sequential selection of economic good and action in medial frontal cortex of macaques during value-based decisions. eLife, 4:e09418, Nov. 2015. ISSN 2050-084X. doi: 10.7554/eLife.09418.

X. Chen and V. Stuphorn. Inactivation of Medial Frontal Cortex Changes Risk Preference. Current Biology, 28(19):3114-3122.e4, Oct. 2018. ISSN 09609822. doi: 10.1016/j.cub.2018.07.043.

G. I. Christopoulos, P. N. Tobler, P. Bossaerts, R. J. Dolan, and W. Schultz. Neural correlates of value, risk, and risk aversion contributing to decision making under risk. Journal of Neuroscience, 29(40):12574-12583, Oct. 2009. ISSN 0270-6474, 1529-2401. doi: 10.1523/JNEUROSCI.2614-09.2009.

E. A. D. Clifton, J. R. B. Perry, F. Imamura, L. A. Lotta, S. Brage, N. G. Forouhi, S. J. Griffin, N. J. Wareham, K. K. Ong, and F. R. Day. Genome-wide association study for risk taking propensity indicates shared pathways with body mass index. Communications Biology, 1(1):36, Dec. 2018. ISSN 2399-3642. doi: 10.1038/s42003-018-0042-6.

L. D. Cohn, S. Macfarlane, C. Yanez, and W. K. Imai. Risk-perception: Differences between adolescents and adults. Health Psychology, 14(3):217-222, 1995. ISSN 1930-7810, 0278-6133. doi: 10.1037/0278-6133.14.3.217.

C. M. Constantinople, A. T. Piet, P. Bibawi, A. Akrami, C. Kopec, and C. D. Brody. Lateral orbitofrontal cortex promotes trial-by-trial learning of risky, but not spatial, biases. eLife, 8:e49744, Nov. 2019a. ISSN 2050-084X. doi: 10.7554/eLife.49744.

C. M. Constantinople, A. T. Piet, and C. D. Brody. An Analysis of Decision under Risk in Rats. Current Biology, 29(12):2066-2074.e5, June 2019b. ISSN 09609822. doi: 10.1016/j.cub.2019.05.013.

M. L. Dent, L. A. Screven, and A. Kobrina. Hearing in Rodents. In M. L. Dent, R. R. Fay, and A. N. Popper, editors, Rodent Bioacoustics, Springer Handbook of Auditory Research, pages 71-105. Springer International Publishing, Cham, 2018. ISBN 978-3-319-92495-3. doi: 10.1007/978-3-319-92495-3_4. 
M. C. Dorris and P. W. Glimcher. Activity in Posterior Parietal Cortex Is Correlated with the Relative Subjective Desirability of Action. Neuron, 44(2):365-378, Oct. 2004. ISSN 08966273. doi: 10.1016/j.neuron.2004.09.009.

D. Duxbury and B. Summers. Financial risk perception. Economics Letters, 84(1): 21-28, July 2004. ISSN 01651765. doi: 10.1016/j.econlet.2003.12.006.

C. L. Ebbesen, M. N. Insanally, C. D. Kopec, M. Murakami, A. Saiki, and J. C. Erlich. More than Just a "Motor": Recent Surprises from the Frontal Cortex. The Journal of Neuroscience, 38(44):9402-9413, Oct. 2018. ISSN 0270-6474, 1529-2401. doi: 10.1523/JNEUROSCI.1671-18.2018.

J. C. Erlich, M. Bialek, and C. D. Brody. A cortical substrate for memory-guided orienting in the rat. Neuron, 72(2):330-343, Oct. 2011. doi:

10.1016/j.neuron.2011.07.010.

J. C. Erlich, B. W. Brunton, C. A. Duan, T. D. Hanks, and C. D. Brody. Distinct effects of prefrontal and parietal cortex inactivations on an accumulation of evidence task in the rat. eLife, 4, 2015. doi: 10.7554/eLife.05457.

J. Essig, J. B. Hunt, and G. Felsen. Inhibitory neurons in the superior colliculus mediate selection of spatially-directed movements. Communications Biology, 4(1): 719, Dec. 2021. ISSN 2399-3642. doi: 10.1038/s42003-021-02248-1.

S. Farashahi, C. H. Donahue, B. Y. Hayden, D. Lee, and A. Soltani. Flexible combination of reward information across primates. Nature Human Behaviour, 3 (11):1215-1224, Nov. 2019. ISSN 2397-3374. doi: 10.1038/s41562-019-0714-3.

S. B. Floresco, D. R. Montes, M. M. Tse, and M. van Holstein. Differential contributions of nucleus accumbens subregions to cue-guided risk/reward decision making and implementation of conditional rules. The Journal of Neuroscience, pages 3191-17, Jan. 2018. ISSN 0270-6474, 1529-2401. doi: 10.1523/JNEUROSCI.3191-17.2018.

M. P. H. Gardner, J. S. Conroy, M. H. Shaham, C. V. Styer, and G. Schoenbaum. Lateral Orbitofrontal Inactivation Dissociates Devaluation-Sensitive Behavior and Economic Choice. Neuron, 96(5):1192-1203.e4, Dec. 2017. ISSN 0896-6273. doi: 10.1016/j.neuron.2017.10.026.

M. J. Goard, G. N. Pho, J. Woodson, and M. Sur. Distinct roles of visual, parietal, and frontal motor cortices in memory-guided sensorimotor decisions. eLife, 5: e13764, Aug. 2016. ISSN 2050-084X. doi: 10.7554/eLife.13764.

T. D. Hanks, C. D. Kopec, B. W. Brunton, C. A. Duan, J. C. Erlich, and C. D. Brody. Distinct relationships of parietal and prefrontal cortices to evidence accumulation. Nature, 520(7546):220-223, Apr. 2015. doi: 10.1038/nature14066.

C. D. Harvey, P. Coen, and D. W. Tank. Choice-specific sequences in parietal cortex during a virtual-navigation decision task. Nature, 484(7392):62-68, Mar. 2012. ISSN 0028-0836, 1476-4687. doi: 10.1038/nature10918.

S. R. Heilbronner. Modeling risky decision-making in nonhuman animals: Shared core features. Current Opinion in Behavioral Sciences, 16:23-29, Aug. 2017. ISSN 2352-1546. doi: 10.1016/j.cobeha.2017.03.001.

D. L. Hocker, C. D. Brody, C. Savin, and C. M. Constantinople. Subpopulations of neurons in $1 \mathrm{OFC}$ encode previous and current rewards at time of choice. eLife, 10: e70129, Oct. 2021. ISSN 2050-084X. doi: 10.7554/eLife.70129. 
S. A. Huettel, C. J. Stowe, E. M. Gordon, B. T. Warner, and M. L. Platt. Neural Signatures of Economic Preferences for Risk and Ambiguity. Neuron, 49(5):765-775, Mar. 2006. ISSN 0896-6273. doi: 10.1016/j.neuron.2006.01.024.

M. N. Insanally, I. Carcea, R. E. Field, C. C. Rodgers, B. DePasquale, K. Rajan, M. R. DeWeese, B. F. Albanna, and R. C. Froemke. Spike-timing-dependent ensemble encoding by non-classically responsive cortical neurons. eLife, 8:e42409, Jan. 2019. ISSN 2050-084X. doi: 10.7554/eLife.42409.

D. Kahneman and A. Tversky. Prospect Theory: An Analysis of Decision under Risk. Econometrica, 47(2):263, Mar. 1979. ISSN 00129682. doi: 10.2307/1914185.

L. N. Katz, J. L. Yates, J. W. Pillow, and A. C. Huk. Dissociated functional significance of decision-related activity in the primate dorsal stream. Nature, 535 (7611):285-288, July 2016. ISSN 0028-0836. doi: 10.1038/nature18617.

K. Kobayashi and M. Hsu. Neural mechanisms of updating under reducible and irreducible uncertainty. Journal of Neuroscience, pages 0535-17, June 2017. ISSN 0270-6474, 1529-2401. doi: 10.1523/JNEUROSCI.0535-17.2017.

K. Kobayashi and M. Hsu. Common neural code for reward and information value. Proceedings of the National Academy of Sciences, 116(26):13061-13066, June 2019. ISSN 0027-8424, 1091-6490. doi: 10.1073/pnas.1820145116.

C. D. Kopec, J. C. Erlich, B. W. Brunton, K. Deisseroth, and C. D. Brody. Cortical and Subcortical Contributions to Short- Term Memory for Orienting Movements. Neuron, 88(2):367-377, Oct. 2015. doi: 10.1016/j.neuron.2015.08.033.

M. Kuwabara, N. Kang, T. E. Holy, and C. Padoa-Schioppa. Neural mechanisms of economic choices in mice. eLife, 9:e49669, Feb. 2020. ISSN 2050-084X. doi: 10.7554/eLife.49669.

J. D. Larkin, N. L. Jenni, and S. B. Floresco. Modulation of risk/reward decision making by dopaminergic transmission within the basolateral amygdala. Psychopharmacology, 233(1):121-136, 2016.

A. M. Licata, M. T. Kaufman, D. Raposo, M. B. Ryan, J. P. Sheppard, and A. K. Churchland. Posterior parietal cortex guides visual decisions in rats. The Journal of neuroscience : the official journal of the Society for Neuroscience, pages 0105-17, Apr. 2017. doi: 10.1523/JNEUROSCI.0105-17.2017.

C. K. Machens. Flexible Control of Mutual Inhibition: A Neural Model of Two-Interval Discrimination. Science, 307(5712):1121-1124, Feb. 2005. ISSN 0036-8075, 1095-9203. doi: 10.1126/science.1104171.

J. G. March. Learning to be risk averse. Psychological Review, 103(2):309-319, Apr. 1996. ISSN 1939-1471, 0033-295X. doi: 10.1037/0033-295X.103.2.309.

I. E. Monosov, D. A. Leopold, and O. Hikosaka. Neurons in the Primate Medial Basal Forebrain Signal Combined Information about Reward Uncertainty, Value, and Punishment Anticipation. Journal of Neuroscience, 35(19):7443-7459, May 2015. ISSN 0270-6474, 1529-2401. doi: 10.1523/JNEUROSCI.0051-15.2015.

A. S. Morcos and C. D. Harvey. History-dependent variability in population dynamics during evidence accumulation in cortex. Nature Neuroscience, 19(12):1672-1681, Dec. 2016. ISSN 1097-6256, 1546-1726. doi: 10.1038/nn.4403. 
Y. Niv, D. Joel, I. Meilijson, and E. Ruppin. Evolution of Reinforcement Learning in Uncertain Environments: A Simple Explanation for Complex Foraging Behaviors. Adaptive Behavior, 10(1):5-24, Jan. 2002. ISSN 1059-7123, 1741-2633. doi: 10.1177/1059-712302-010001-01.

J. P. O'Doherty, S. W. Lee, and D. McNamee. The structure of reinforcement-learning mechanisms in the human brain. Current Opinion in Behavioral Sciences, 1:94-100, Feb. 2015. ISSN 23521546. doi: 10.1016/j.cobeha.2014.10.004.

M. Ogawa, M. A. A. van der Meer, G. R. Esber, D. H. Cerri, T. A. Stalnaker, and G. Schoenbaum. Risk-Responsive Orbitofrontal Neurons Track Acquired Salience. Neuron, 77(2):251-258, Jan. 2013. ISSN 0896-6273. doi: 10.1016/j.neuron.2012.11.006.

C. A. Orsini, D. E. Moorman, J. W. Young, B. Setlow, and S. B. Floresco. Neural mechanisms regulating different forms of risk-related decision-making: Insights from animal models. Neuroscience \& Biobehavioral Reviews, 58:147-167, Nov. 2015. ISSN 0149-7634. doi: 10.1016/j.neubiorev.2015.04.009.

C. A. Orsini, C. M. Hernandez, S. Singhal, K. B. Kelly, C. J. Frazier, J. L. Bizon, and B. Setlow. Optogenetic inhibition reveals distinct roles for basolateral amygdala activity at discrete timepoints during risky decision making. Journal of Neuroscience, pages 2344-17, Oct. 2017. ISSN 0270-6474, 1529-2401. doi: 10.1523/JNEUROSCI.2344-17.2017.

C. Padoa-Schioppa. Neurobiology of Economic Choice: A Good-Based Model. Annual Review of Neuroscience, 34(1):333-359, July 2011. ISSN 0147-006X, 1545-4126. doi: 10.1146/annurev-neuro-061010-113648.

A. T. Piet, J. C. Erlich, C. D. Kopec, and C. D. Brody. Rat Prefrontal Cortex Inactivations during Decision Making Are Explained by Bistable Attractor Dynamics. Neural Computation, 29(11):2861-2886, Aug. 2017. ISSN 0899-7667. doi: 10.1162/neco_a_01005.

M. L. Platt and P. W. Glimcher. Neural correlates of decision variables in parietal cortex. Nature, 400(6741):233-238, July 1999. ISSN 0028-0836, 1476-4687. doi: $10.1038 / 22268$.

M. L. Platt and S. A. Huettel. Risky business: The neuroeconomics of decision making under uncertainty. Nature Neuroscience, 11(4):398-403, Apr. 2008. ISSN 1097-6256, 1546-1726. doi: 10.1038/nn2062.

M. Rabin and R. H. Thaler. Anomalies: Risk Aversion. Journal of Economic Perspectives, 15(1):219-232, Feb. 2001. ISSN 0895-3309. doi: 10.1257/jep.15.1.219.

L.-L. Rao, Y. Zhou, D. Zheng, L.-Q. Yang, and S. Li. Genetic Contribution to Variation in Risk Taking: A Functional MRI Twin Study of the Balloon Analogue Risk Task. Psychological Science, 29(10):1679-1691, Oct. 2018. ISSN 0956-7976, 1467-9280. doi: 10.1177/0956797618779961.

D. Raposo, M. T. Kaufman, and A. K. Churchland. A category-free neural population supports evolving demands during decision-making. Nature Neuroscience, 17(12): 1784-1792, Dec. 2014. ISSN 1546-1726. doi: 10.1038/nn.3865.

R. L. Reep and J. V. Corwin. Posterior parietal cortex as part of a neural network for directed attention in rats. Neurobiology of Learning and Memory, 91(2):104-113, Feb. 2009. ISSN 1074-7427. doi: 10.1016/j.nlm.2008.08.010. 
M. R. Roesch, A. R. Taylor, and G. Schoenbaum. Encoding of Time-Discounted Rewards in Orbitofrontal Cortex Is Independent of Value Representation. Neuron, 51(4):509-520, Aug. 2006. ISSN 0896-6273. doi: 10.1016/j.neuron.2006.06.027.

R. A. Saez, A. Saez, J. J. Paton, B. Lau, and C. D. Salzman. Distinct Roles for the Amygdala and Orbitofrontal Cortex in Representing the Relative Amount of Expected Reward. Neuron, 95(1):70-77.e3, July 2017. ISSN 0896-6273. doi: 10.1016/j.neuron.2017.06.012.

K. Samejima, Y. Ueda, K. Doya, and M. Kimura. Representation of action-specific reward values in the striatum. Science (New York, N.Y.), 310(5752):1337-1340, Nov. 2005. ISSN 1095-9203. doi: 10.1126/science.1115270.

B. B. Scott, C. M. Constantinople, J. C. Erlich, D. W. Tank, and C. D. Brody. Sources of noise during accumulation of evidence in unrestrained and voluntarily head-restrained rats. eLife, 4:e11308, Dec. 2015. doi: 10.7554/eLife.11308.

B. B. Scott, C. M. Constantinople, A. Akrami, T. D. Hanks, C. D. Brody, and D. W. Tank. Fronto-parietal Cortical Circuits Encode Accumulated Evidence with a Diversity of Timescales. Neuron, June 2017. ISSN 0896-6273. doi: 10.1016/j.neuron.2017.06.013.

J. Shires, S. Joshi, and M. A. Basso. Shedding new light on the role of the basal ganglia-superior colliculus pathway in eye movements. Current Opinion in Neurobiology, 20(6):717-725, Dec. 2010. ISSN 09594388. doi: 10.1016/j.conb.2010.08.008.

M. J. Siniscalchi, V. Phoumthipphavong, F. Ali, M. Lozano, and A. C. Kwan. Fast and slow transitions in frontal ensemble activity during flexible sensorimotor behavior. Nature Neuroscience, 19(9):1234-1242, Sept. 2016. ISSN 1097-6256, 1546-1726. doi: 10.1038/nn.4342.

M. Spitmaan, E. Chu, and A. Soltani. Salience-Driven Value Construction for Adaptive Choice under Risk. The Journal of Neuroscience, 39(26):5195-5209, June 2019. ISSN 0270-6474, 1529-2401. doi: 10.1523/JNEUROSCI.2522-18.2019.

C. M. Stopper, E. B. Green, and S. B. Floresco. Selective involvement by the medial orbitofrontal cortex in biasing risky, but not impulsive, choice. Cerebral cortex, 24 (1):154-162, 2012.

C. M. Stopper, S. Khayambashi, and S. B. Floresco. Receptor-Specific Modulation of Risk-Based Decision Making by Nucleus Accumbens Dopamine. Neuropsychopharmacology, 38(5):715-728, Apr. 2013. ISSN 0893-133X. doi: 10.1038/npp.2012.240.

The International Brain Laboratory, V. Aguillon-Rodriguez, D. Angelaki, H. Bayer, N. Bonacchi, M. Carandini, F. Cazettes, G. Chapuis, A. K. Churchland, Y. Dan, E. Dewitt, M. Faulkner, H. Forrest, L. Haetzel, M. Häusser, S. B. Hofer, F. Hu, A. Khanal, C. Krasniak, I. Laranjeira, Z. F. Mainen, G. Meijer, N. J. Miska, T. D. Mrsic-Flogel, M. Murakami, J.-P. Noel, A. Pan-Vazquez, C. Rossant, J. Sanders, K. Socha, R. Terry, A. E. Urai, H. Vergara, M. Wells, C. J. Wilson, I. B. Witten, L. E. Wool, and A. M. Zador. Standardized and reproducible measurement of decision-making in mice. eLife, 10:e63711, May 2021. ISSN 2050-084X. doi: 10.7554/eLife.63711. 
M. van Holstein and S. B. Floresco. Dissociable roles for the ventral and dorsal medial prefrontal cortex in cue-guided risk/reward decision making. Neuropsychopharmacology, 45(4):683-693, Mar. 2020. ISSN 0893-133X, 1740-634X. doi: $10.1038 / \mathrm{s} 41386-019-0557-7$.

J. Von Neumann and O. Morgenstern. Theory of Games and Economic Behavior. Princeton University press, Princeton (N.J.), 3rd ed edition, 1953. ISBN 978-0-691-04183-4.

E. U. Weber, S. Shafir, and A.-R. Blais. Predicting Risk Sensitivity in Humans and Lower Animals: Risk as Variance or Coefficient of Variation. Psychological Review, 111(2):430-445, 2004. ISSN 1939-1471, 0033-295X. doi: 10.1037/0033-295X.111.2.430.

A. F. Williams. Teenage drivers: Patterns of risk. Journal of Safety Research, 34(1): 5-15, Jan. 2003. ISSN 00224375. doi: 10.1016/S0022-4375(02)00075-0.

Y.-H. Xuan, S. Li, R. Tao, J. Chen, L.-L. Rao, X. T. Wang, and R. Zheng. Genetic and Environmental Influences on Gambling: A Meta-Analysis of Twin Studies. Frontiers in Psychology, 8:2121, Dec. 2017. ISSN 1664-1078. doi: 10.3389/fpsyg.2017.02121.

J. F. Yates, editor. Risk-Taking Behavior. Wiley Series, Human Performance and Cognition. Wiley, Chichester, West Sussex, England ; New York, 1992. ISBN 978-0-471-92250-6.

K. A. Zalocusky, C. Ramakrishnan, T. N. Lerner, T. J. Davidson, B. Knutson, and K. Deisseroth. Nucleus accumbens D2R cells signal prior outcomes and control risky decision-making. Nature, 531(7596):642-646, Mar. 2016. ISSN 0028-0836. doi: $10.1038 /$ nature17400.

L. Zhong, Y. Zhang, C. A. Duan, J. Deng, J. Pan, and N.-l. Xu. Causal contributions of parietal cortex to perceptual decision-making during stimulus categorization.

Nature Neuroscience, 22(6):963-973, June 2019. ISSN 1546-1726. doi: 10.1038/s41593-019-0383-6. 

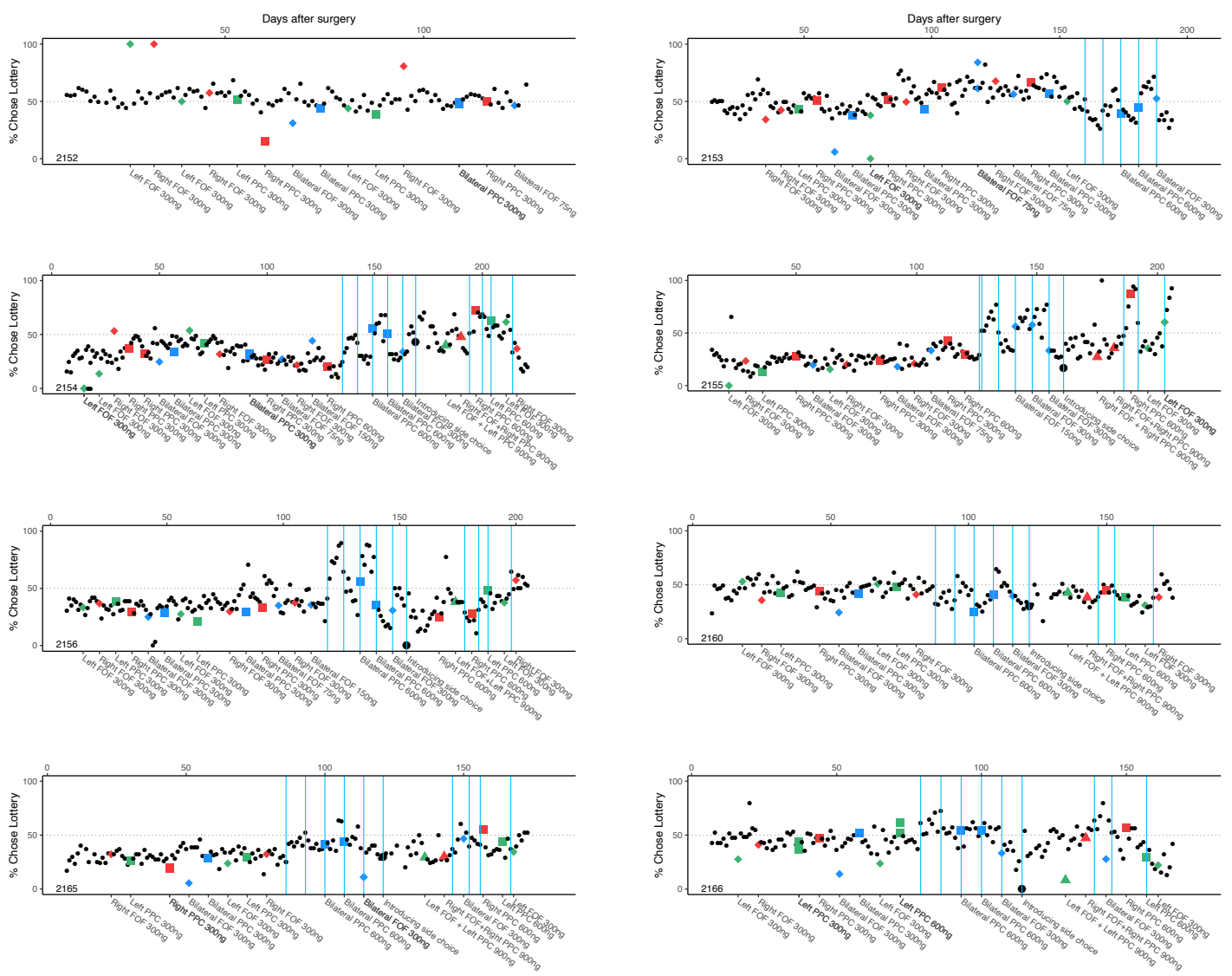

Figure S1. Timeline of percentage choosing the lottery in each session for each rat and a visual summary of the experimental treatments. Each point is the percentage choosing lottery for the given session. The number at the $\mathrm{x}$-axis indicates the days passed since the surgical implantation of cannulae. Control days are shown as small black dots. Right infusions are shown in red, left infusions are in green, and bilateral infusions are shown in blue. FOF infusions are represented by diamonds, PPC infusions by squares, both FOF and PPC infusions by triangles. The blue bars indicate the day of a model-based surebet value change. The large black dot indicates the day when free choice trials were introduced. The bottom x-labels describe the details (side, region and dose) of each infusion. 


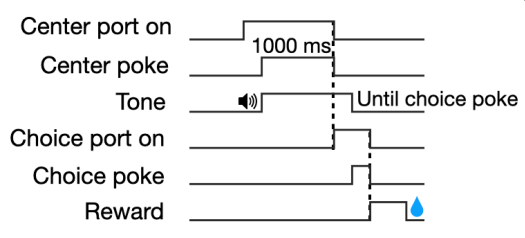

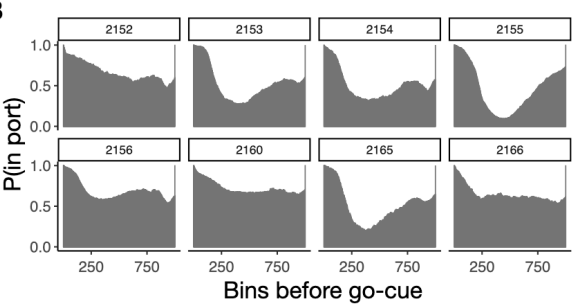

Figure S2. Task and behavior. A. Illustration of the timeline of a risky choice trial. B. The probability of being in the center port before the go-cue and after the first center port poke. For each trial, the period between the initial poke and the go-cue was segmented into 1000 bins. Go-cue is defined as the onset of the choice port lights. For each bin, a binary value was obtained to indicate whether the animal was in the port or not. The probability of being in the port for each bin was calculated by taking the mean of the binary vector. Only control trials were used for this analysis.

A

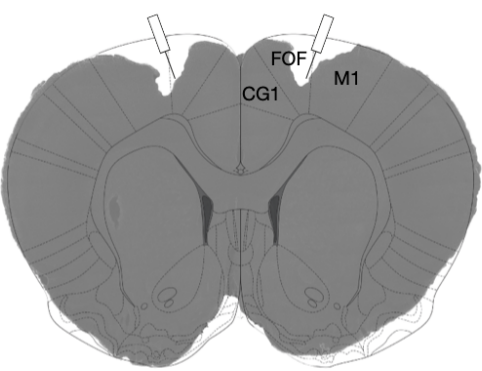

c

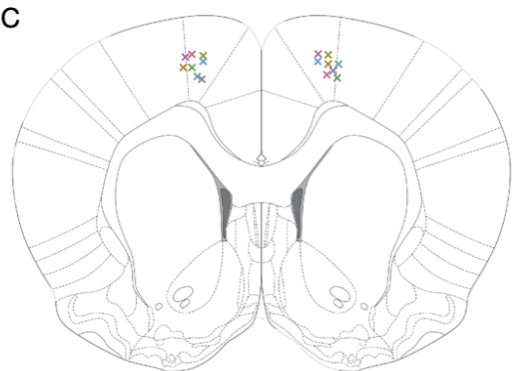

B

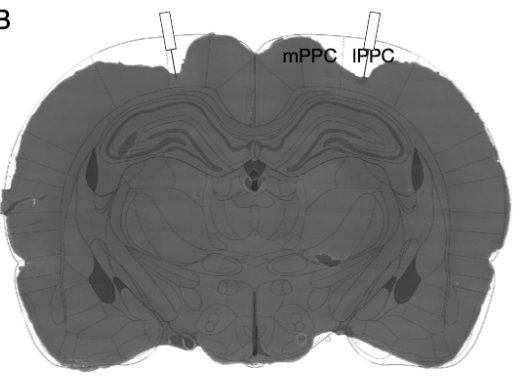

D

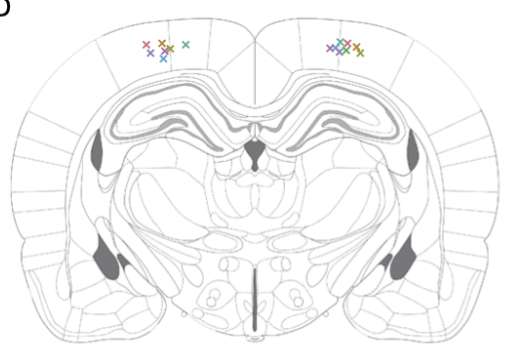

Figure S3. Histology. A. Coronal section of an example rat brain showing cannulae implanted at $20^{\circ}$ in FOF, overlaid with a section $2.04 \mathrm{~mm}$ anterior to Bregma (Paxinos and Watson, 2004). Note, that in the nomenclature of Paxinos and Watson (2004) the area that we describe as the FOF is considered to be part of M2. CG1 = Cingulate Cortex. B. Coronal section of an example rat brain showing cannulae implanted at $10^{\circ}$ in $\mathrm{PPC}$, overlaid with a section 3.48 $\mathrm{mm}$ posterior to Bregma. $\mathrm{mPPC}=$ medial $\mathrm{PPC}, \mathrm{IPPC}=$ lateral PPC. C. Actual cannulae placements in FOF, color represents the subject ID as in FIGURE 1E. D. Cannulae placements in PPC. 
A

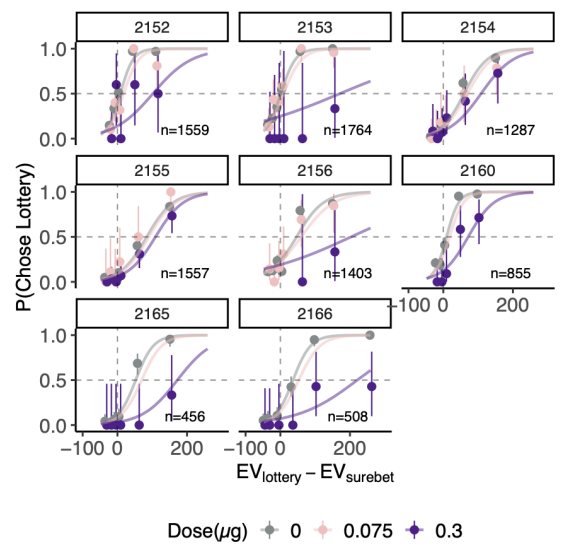

B

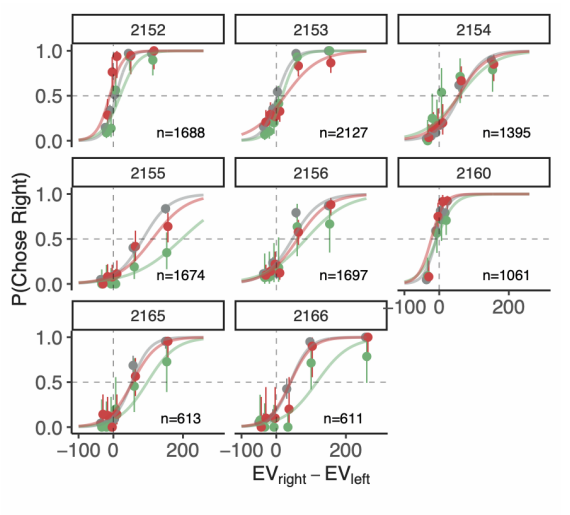

Infusion Side + Control $+\mathrm{L}+\mathrm{R}$

Figure S4. FOF inactivations. The circles with error bars are the binned mean and $95 \%$ binomial confidence intervals. The lines are the model predictions generated by the GLMM. A. Bilateral FOF inactivation with $0.075 \mu \mathrm{g}$ and $0.3 \mu \mathrm{g}$ muscimol per side. B. Unilateral FOF inactivation with $0.3 \mu \mathrm{g}$ muscimol.

A

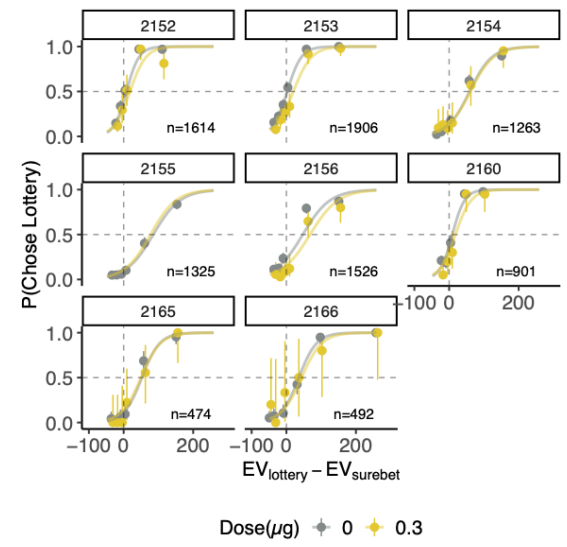

B

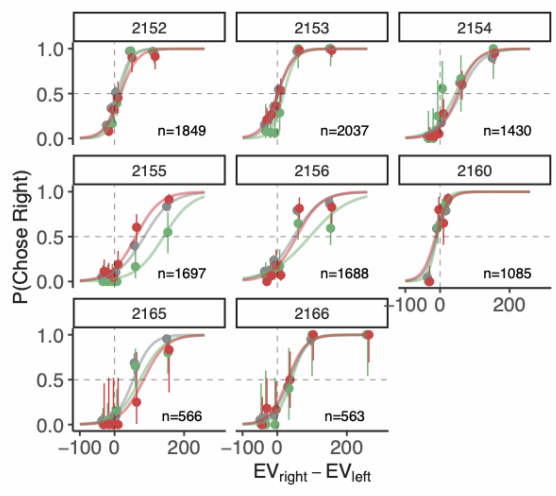

Infusion Side $\phi$ Control $\phi \mathbf{L}+\mathrm{R}$

Figure S5. PPC inactivations. The circles with error bars are the binned mean and $95 \%$ binomial confidence intervals. The lines are the model predictions generated by the GLMM. A. Bilateral PPC inactivation with $0.3 \mu \mathrm{g}$ muscimol per side. B. Unilateral PPC inactivation with $0.3 \mu \mathrm{g}$ muscimol. 
A

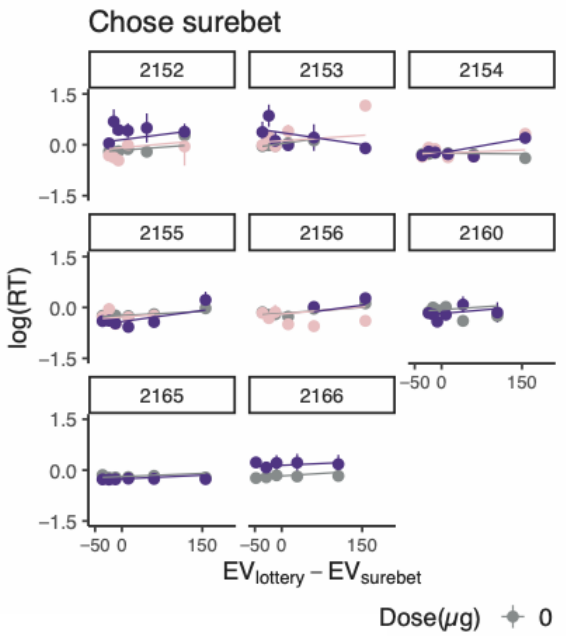

B

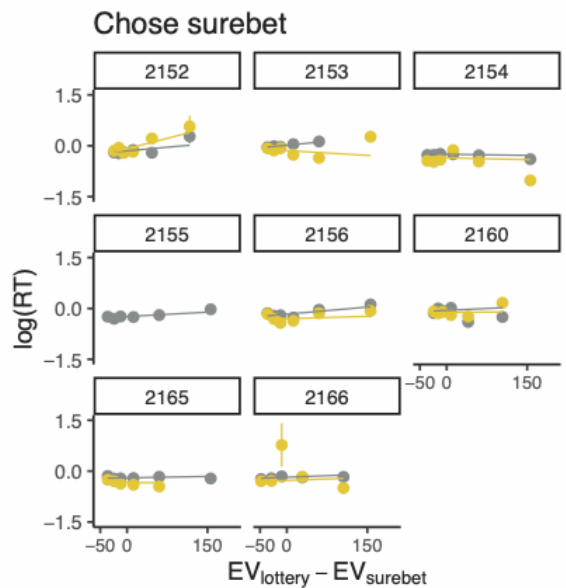

Bilateral PPC
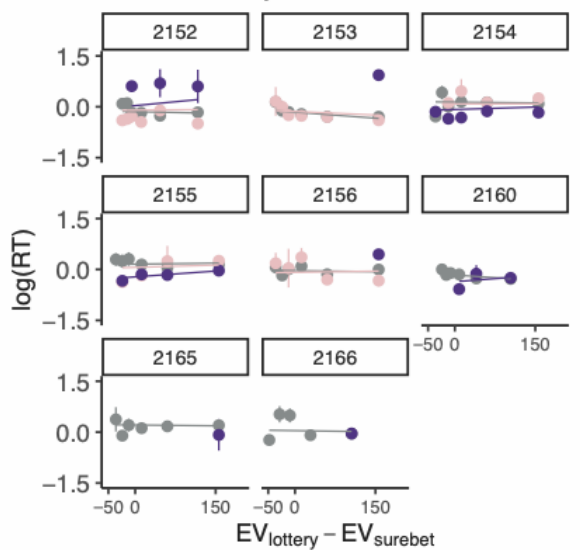

$0.075+0.3$

$$
\operatorname{Dose}(\mu \mathrm{g})+0+0.3
$$

Figure S6. Reaction time (RT) and LMM model fits for bilateral FOF and PPC inactivations. The circles with error bars represent the mean and standard error of $\log (\mathrm{RT})$. The lines are the model predictions generated by the LMM. A. Bilateral FOF inactivation. Reaction times are from the same trials as presented in FiguRE S4A. B. Bilateral PPC inactivation. Reaction times are from the same trials as presented in FigurE S5A. 
A

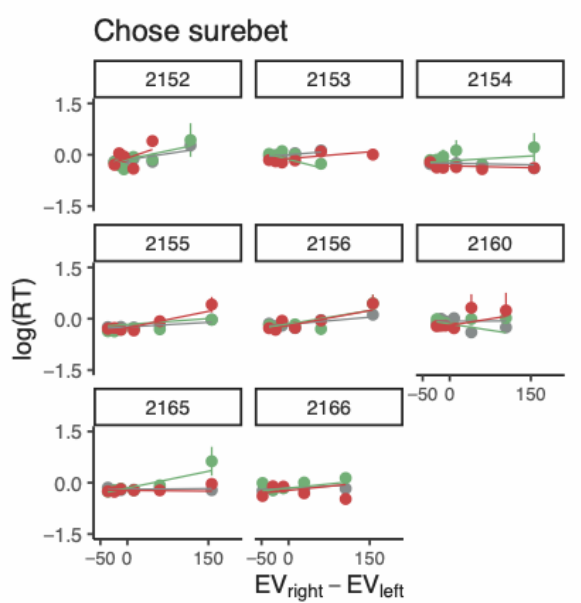

Unilateral FOF

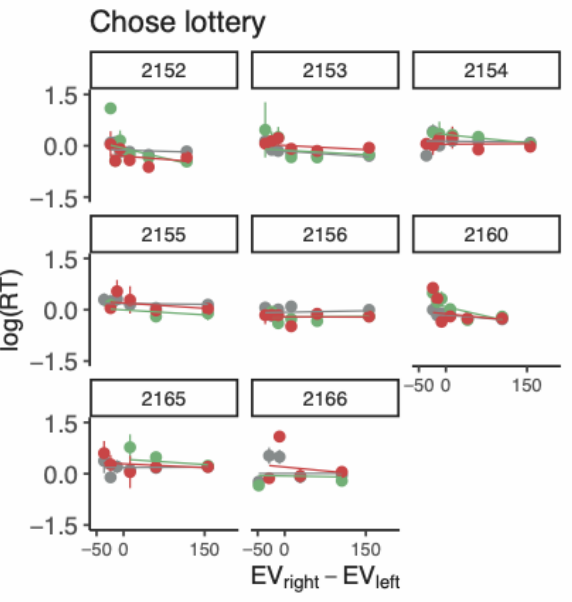

Infusion Side $\phi$ Control $+\mathrm{L}+\mathrm{R}$

B

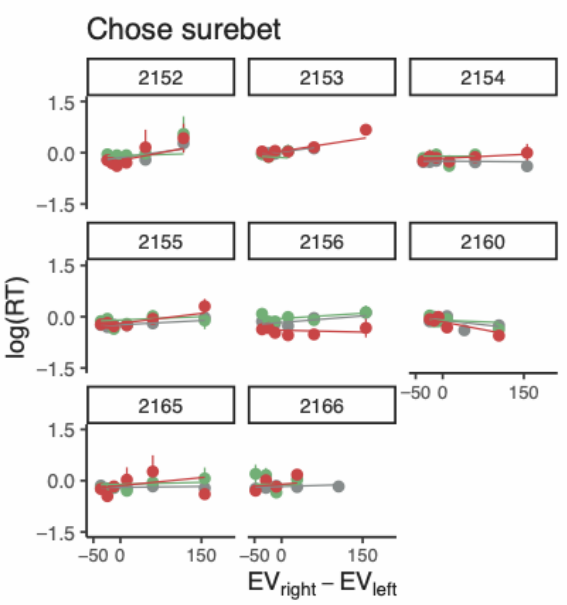

Unilateral PPC

\section{Chose lottery}
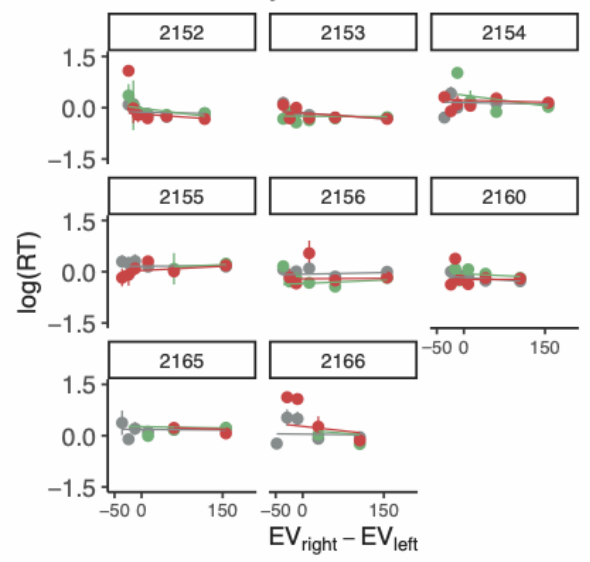

Infusion Side $\$$ - Control $+\mathrm{L}-\mathrm{R}$

Figure S7. RT and LMM model fits for unilateral FOF and PPC inactivation trials. The circles with error bars represent the mean and standard error of $\log (\mathrm{RT})$. The lines are the model predictions generated by the LMM. A. Bilateral FOF inactivation. Reaction times are from the same trials as presented in FigurE S4B. B. Bilateral PPC inactivation. Reaction times are from the same trials as presented in FigurE S5B. 
A

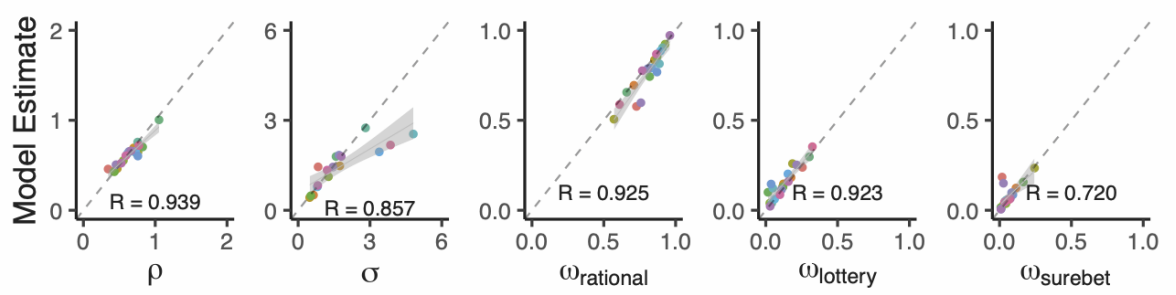

B
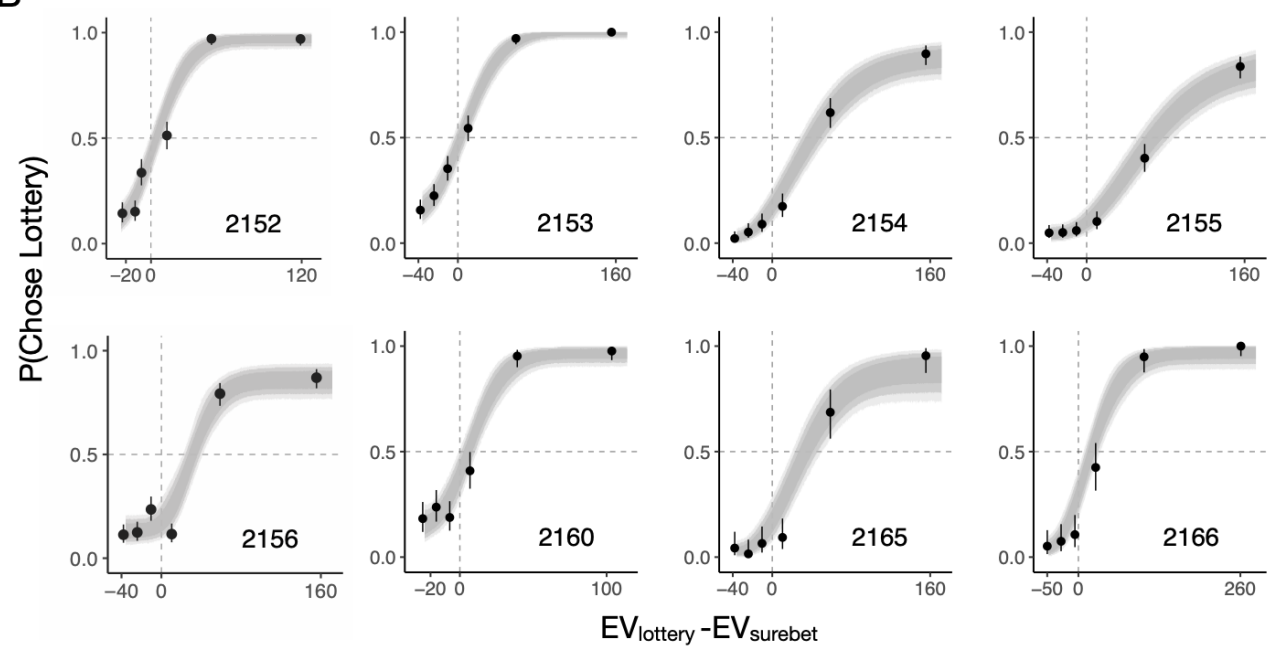

Figure S8. The three-agent mixture model. A. The model can recover the data-generating parameters well. Twenty Synthetic datasets were created by sampling from the same prior distributions as specified in Methods. The true parameter value is on the $\mathrm{X}$-axis, the maximum a posteriori model estimation is on the Y-axis. Color represents the identity of each synthetic dataset. All the parameters fall along the diagonal line. B. The psychometric data and model prediction from the three-agent mixture model for 8 animals. The circles with error bars are the binned mean and $95 \%$ binomial confidence intervals. The ribbons are model predictions generated using the fitted parameters. The dark, medium and light shade represent $80 \%, 95 \%$ and $99 \%$ confidence intervals, respectively. Data used are the same as the control sessions in FiguRE $1 \mathrm{C}$. 

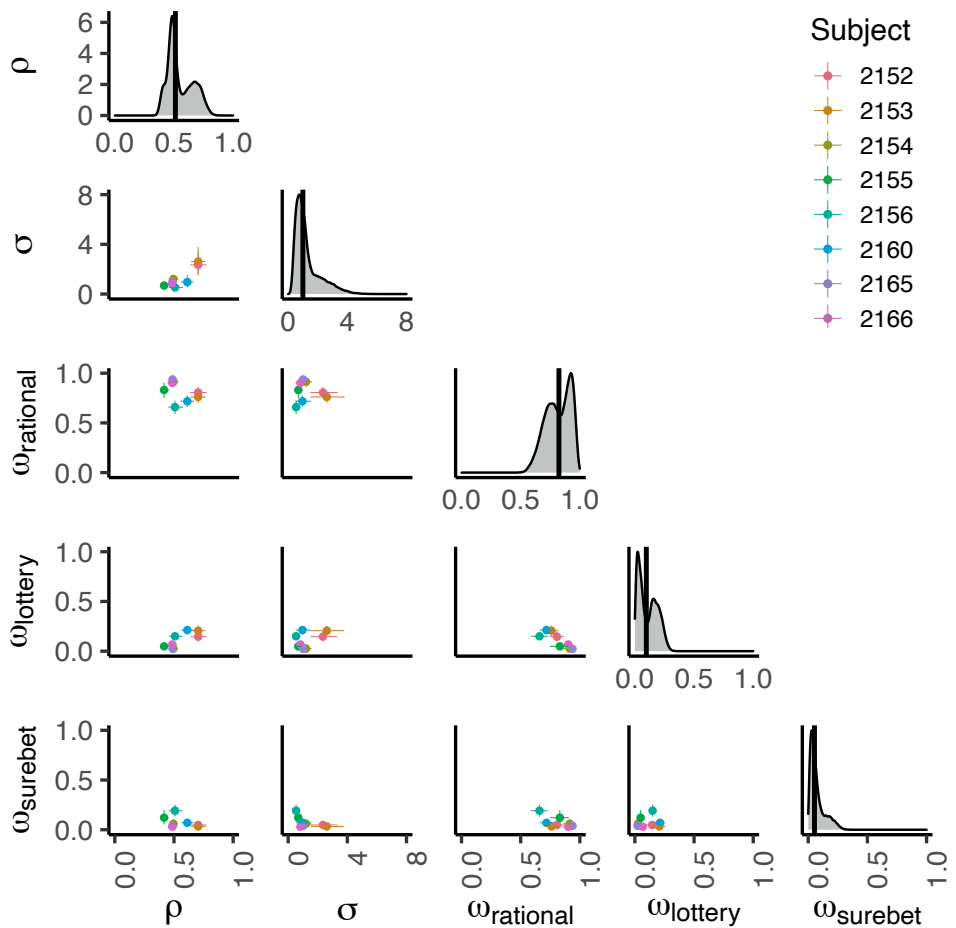

Figure S9. Summary of the fit model parameters from the control sessions of 8 animals. The mean and $95 \%$ confidence interval of each parameter pair are shown in the off-diagonal, colored by subject. Density plots of concatenated posterior samples for each parameter are on the diagonal, the black bar denotes the median.
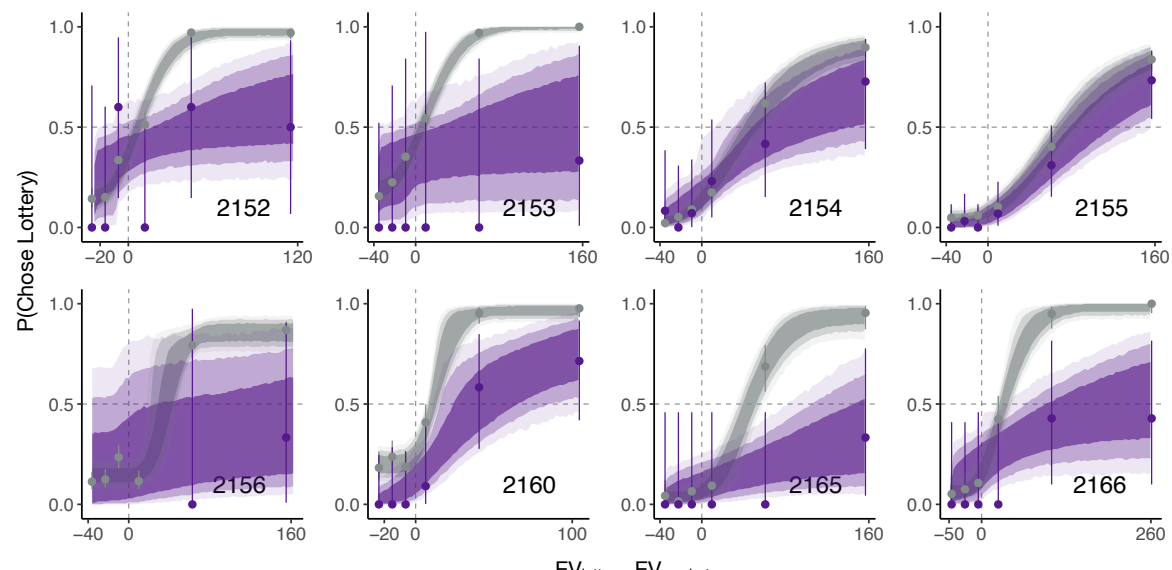

Figure S10. Subjects' choices superimposed with the inactivation model fit on control (in gray) and bilateral FOF inactivation (in purple) dataset simultaneously. The circles with error bars are the binned mean and $95 \%$ binomial confidence intervals. The ribbons are model predictions generated using the fitted parameters. The dark, medium and light shade represent $80 \%, 95 \%$ and $99 \%$ confidence intervals, respectively. 
2152

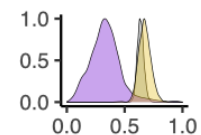

2153

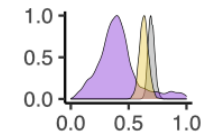

2154

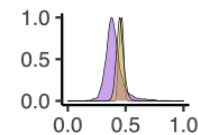

2155

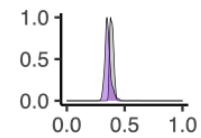

2156

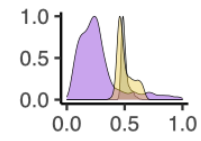

2160

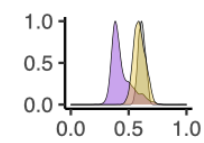

2165

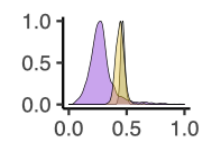

2166

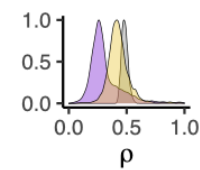

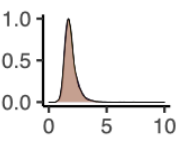
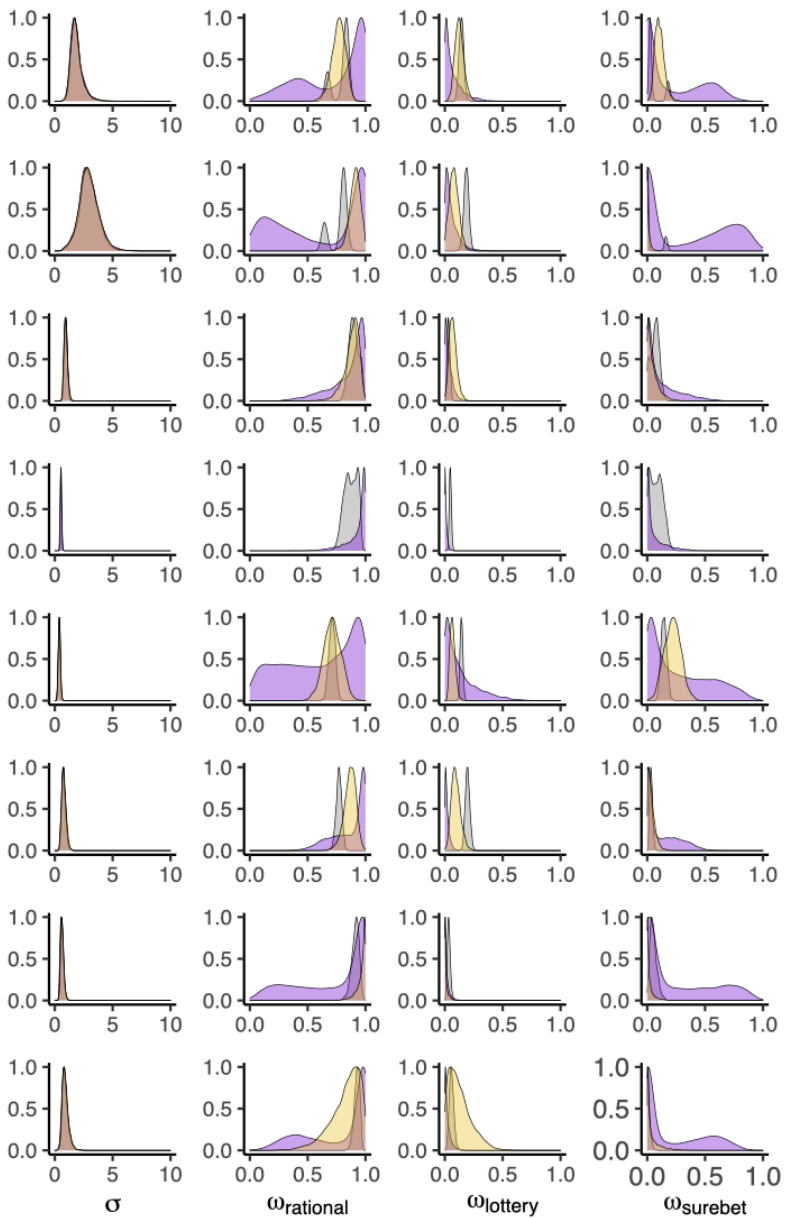

Figure S11. Posterior distributions for each parameter using the inactivation model fit to the control (in gray), $0.3 \mu \mathrm{g}$ per side bilateral FOF inactivation (in purple) and $0.3 \mu \mathrm{g}$ per side bilateral PPC inactivation (in gold) dataset simultaneously. To allow easier visual comparison, all posteriors were normalized so that the peak of the distribution was set to 1 . Since subject 2155 lost one PPC cannula, only the control and bilateral FOF fit was included here. From left to right: $\rho$ is the exponent on the utility function, $\sigma$ denotes the noise in utility representation, $\omega_{\text {rational }}$ is the weight of the rational agent, $\omega_{\text {lottery }}$ is the weight of the lottery agent, and $\omega_{\text {surebet }}$ is the weight of the surebet agent. 

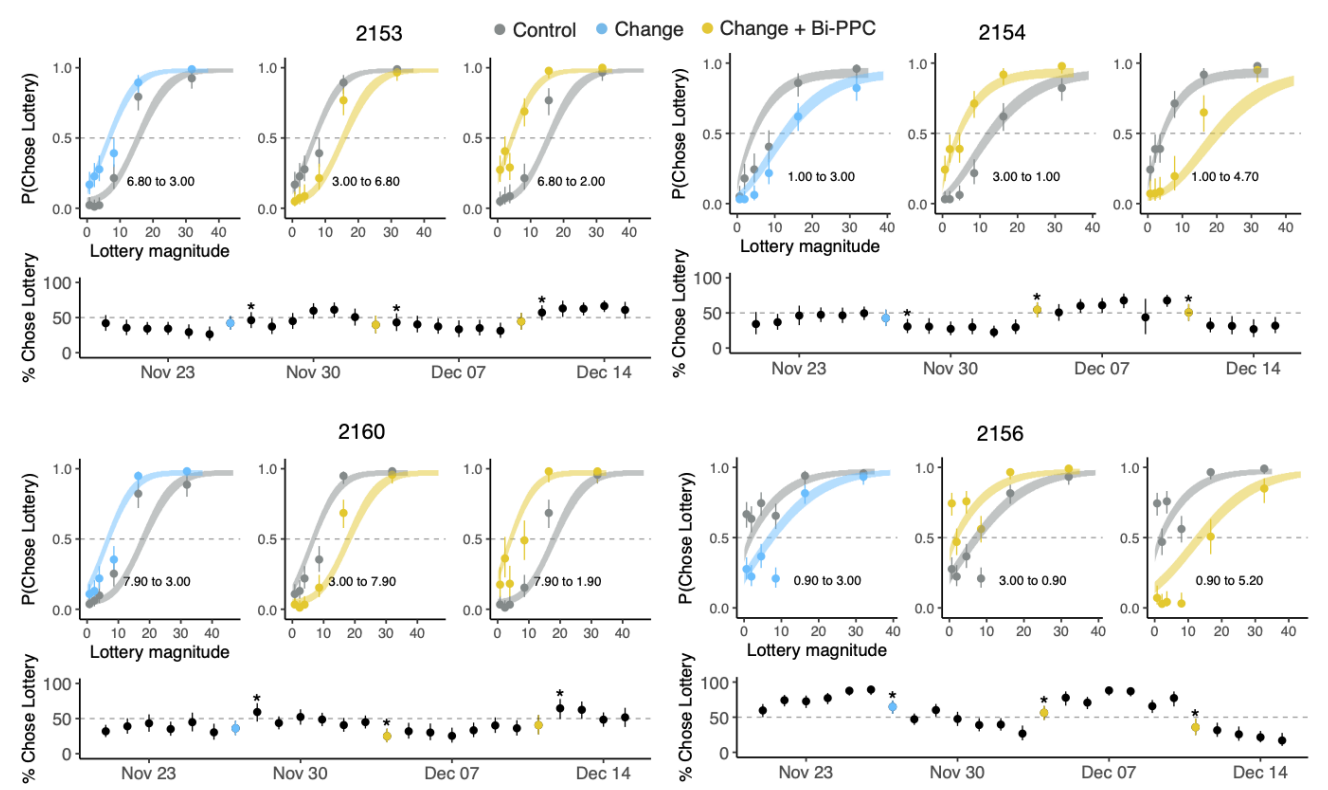

Figure S12. Behavioral adaptation of subject 2153, 2154, 2156 and 2160 in the surebet learning experiment. Only one model was fit to all the trials and used for prediction for each animal. Top three subpanels: the circles with error bars are the binned mean and $95 \%$ binomial confidence intervals; the ribbons are generated using the fit parameter posterior of with $80 \%$ confidence intervals. Behavior from 6 sessions immediately before a surebet change is in gray, behavior from 7 sessions after a surebet change (including the very day) is in light blue if no infusion, in gold if with $0.6 \mu \mathrm{g}$ bilateral PPC infusion. Text annotation shows the old and new surebet magnitudes. Bottom subpanel: The percentage choosing lottery of each session. Asterisk indicates when change in choices can be significantly detected on that session compared to the previous 6 sessions with old surebet magnitude. 